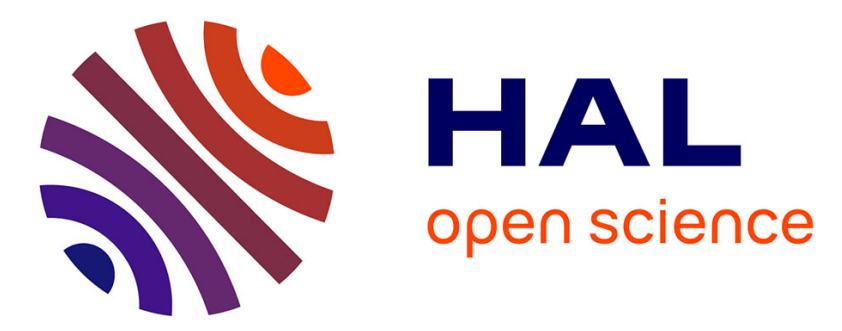

\title{
Steady state analysis of a syntrophic model: the effect of a new input substrate concentration
}

\author{
Yessmine Daoud, Nahla Abdellatif, Tewfik Sari, Jérome Harmand
}

\section{To cite this version:}

Yessmine Daoud, Nahla Abdellatif, Tewfik Sari, Jérome Harmand. Steady state analysis of a syntrophic model: the effect of a new input substrate concentration. Mathematical Modelling of Natural Phenomena, 2018, 13 (3), pp.21. 10.1051/mmnp/2018037 . hal-02608022

\section{HAL Id: hal-02608022 \\ https://hal.inrae.fr/hal-02608022}

Submitted on 16 May 2020

HAL is a multi-disciplinary open access archive for the deposit and dissemination of scientific research documents, whether they are published or not. The documents may come from teaching and research institutions in France or abroad, or from public or private research centers.
L'archive ouverte pluridisciplinaire HAL, est destinée au dépôt et à la diffusion de documents scientifiques de niveau recherche, publiés ou non, émanant des établissements d'enseignement et de recherche français ou étrangers, des laboratoires publics ou privés.

$$
\text { Copyright }
$$




\title{
STEADY STATE ANALYSIS OF A SYNTROPHIC MODEL: THE EFFECT OF A NEW INPUT SUBSTRATE CONCENTRATION
}

\author{
Y. Daoud ${ }^{1,2, *}$, N. Abdellatif ${ }^{1,5}$, T. Sari ${ }^{3,6}$ And J. Harmand ${ }^{4}$
}

\begin{abstract}
In this work, we are interested in a reduced and simplified model of the anaerobic digestion process. We focus on the acetogenesis and hydrogenetrophic methanogenesis phases. The model describes a syntrophic relashionship between two microbial species (the acetogenic bacteria and the hydrogenetrophic methanogenic bacteria) with two input substrates (the fatty acids and the hydrogen) including both decay terms and inhibition of the acetogenic bacteria growth by an excess of hydrogen in the system. The existence and stability analysis of the steady states of the model points out the existence of a new equilibrium point which can be stable according to the operating parameters of the system. By means of operating diagrams, we show that, whatever the region of space considered, there exists only one locally exponentially stable steady state.
\end{abstract}

Mathematics Subject Classification. 34D20, 92D25, 92C45

Accepted March 9, 2018.

\section{INTRODUCTION}

The anaerobic digestion (AD) is a natural process in which organic material is converted into biogas in an environment without oxygen by the action of a microbial ecosystem. It is used for the treatment of waste or wastewater and has the advantage of producing methane or hydrogen under appropriate conditions. Thus, it has a high potential within the actual context of green energy development. However, its management is not easy because a number of intermediate metabolites may accumulate and lead to the destabilization of the biological reactions. To better understand and control this process, many models have been reported in the literature, $c f .[1-4,6,8,10,11]$. In particular, a key biological step has been described as the syntrophic relationship between acid consumers (which produce hydrogen) and hydrogen consumer (which produce methane). Indeed, in degrading the hydrogen - which is inhibiting microbial growth rate - methanogens allow their coexistence with acid producers: this fragile equilibrium has been thoroughly studied in the past years. In [11], a model of such a syntrophic relationship is studied. As underlined in this paper, for thermodynamic reasons propionate degradation is extremely sensitive to accumulation of hydrogen. Thus in

Keywords and phrases: Microbial ecosystems, syntrophic relationship, mortality, stability, operating diagrams.

${ }^{1}$ Université de Tunis El Manar, École Nationale d'Ingénieurs de Tunis, LAMSIN, BP 37, Le Belvédère, 1002 Tunis, Tunisie.

${ }^{2}$ UMR Mistea, INRA, Université de Montpellier, 34060 Montpellier, France.

3 UMR Itap, Irstea, Université de Montpellier, 34196 Montpellier, France.

${ }^{4}$ LBE, INRA, Université de Montpellier, 11100 Narbonne, France.

5 Université de Manouba, ENSI, Campus Universitaire de Manouba, 2010 Manouba, Tunisie.

${ }^{6}$ Université de Haute Alsace, LMIA, 4 rue des frères Lumière, 68093 Mulhouse, France.

* Corresponding author: daoud_yessmine@yahoo.fr 


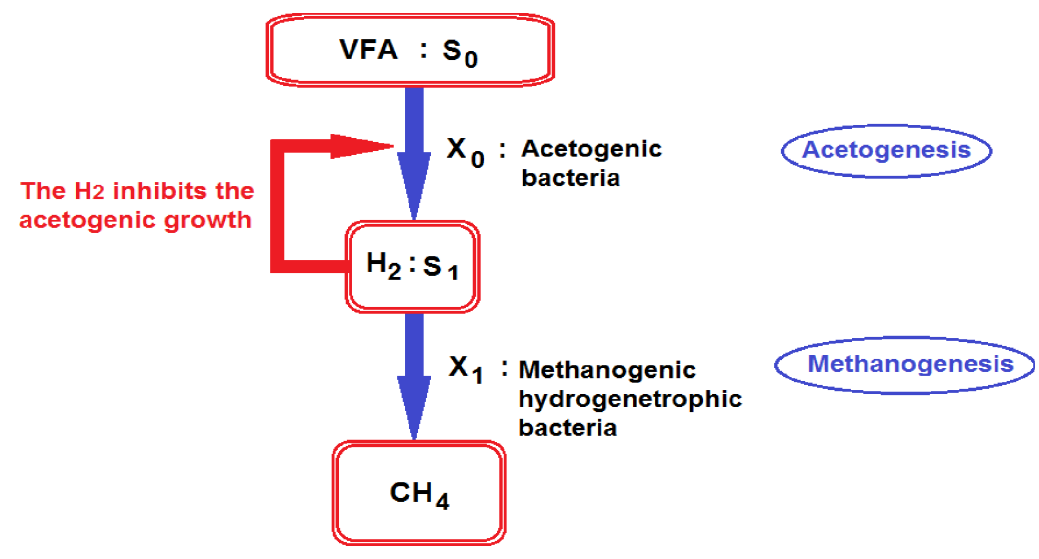

FIGURE 1. The acetogenesis and the hydrogenetrophic methanogenesis phases: the fatty acids produced by the previous phase (the acidogenesis) are consumed by the acetogenic bacteria to produce the hydrogen, which is converted by the hydrogenetrophic methanogenic bacteria into methane. An excess of hydrogen in the system can inhibit the acetogenic bacteria growth.

methanogenic ecosystems propionate degradation is only sustainable in the presence of hydrogenotrophic organisms. To study the syntrophy, the authors have considered a system involving precisely propionate degraders and hydrogenotrophic methanogens. The substrate/product variables are the propionate and the hydrogen (cf. Fig. 1).

Using realistic parameters values for this two-step model, $\mathrm{Xu}$ et al. (cf. [11]) have shown that the introduction of maintenance terms (equivalent to mortality terms in their study) does not destabilize the positive equilibrium of the system. This result has been made generic by Sari and Harmand (cf. [5]) in the sense they have shown that for a large class of kinetics and whatever the model parameters values, the stability of the equilibrium is maintained. However, in these studies, only one substrate input - the input substrate concentration in propionate - was considered. In reality, some hydrogen is produced by other reactions taking place in parallel of the main reactions considered in the model under interest. Thus, to deal with a more realistic situation, we incorporate the input substrate concentration in hydrogen in the model. The aim of this study is to give a comprehensive analysis of the extended model of [5]. We describe all steady states of the model and their stability. We prove, in particular, that the existence of the steady state, corresponding to the washout of acetogenic bacteria, is possible for certain values of the operating parameters and we give necessary and sufficient conditions for its stability. To describe the qualitative behavior of the system, we determine the operating diagram of the model according to the the operating parameters. The operating diagrams can be useful to interpret experimental results. With respect to purely commensalistic systems described by [7], our model is different because of the dependence of the growth rate of microorganisms of the first step by the product of the reaction. With respect to more general models as those considered in [2] or [6], it differs in that it includes mortality terms while the latter do not.

The paper is organized as follows. In Section 2, we present the two-step model with two input substrate concentrations and we give a preliminary result on the positivity and the boundedness of the solution under general hypotheses on the growth functions. In Section 3, we give the description of the steady states and in section 4, we discuss their stability. In Section 5, we illustrate the effect of the second input substrate concentration, in designing the operating diagrams, first, with respect to the first input substrate concentration and the dilution rate and second, with respect to the second input substrate concentration and the dilution rate. In Section 6, numerical simulations with realistic growth functions are presented to illustrate our results in different cases. The technical proofs of the results are given in the Appendix A. 


\section{THE MODEL}

The two-step model reads:

$$
\left\{\begin{aligned}
\frac{d s_{0}}{d t} & =D\left(s_{0}^{i n}-s_{0}\right)-\mu_{0}\left(s_{0}, s_{1}\right) x_{0}, \\
\frac{d x_{0}}{d t} & =-D x_{0}+\mu_{0}\left(s_{0}, s_{1}\right) x_{0}-a_{0} x_{0}, \\
\frac{d s_{1}}{d t} & =D\left(s_{1}^{i n}-s_{1}\right)+\mu_{0}\left(s_{0}, s_{1}\right) x_{0}-\mu_{1}\left(s_{1}\right) x_{1}, \\
\frac{d x_{1}}{d t} & =-D x_{1}+\mu_{1}\left(s_{1}\right) x_{1}-a_{1} x_{1},
\end{aligned}\right.
$$

where $s_{0}$ and $s_{1}$ are the concentration substrates (the fatty acid and the hydrogen, respectively), introduced in the chemostat with input concentrations $s_{0}^{i n}$ and $s_{1}^{i n}$. D is the dilution rate, $x_{0}$ and $x_{1}$ are the acetogenic bacteria and hydrogenetrophic methanogenic bacteria concentrations. This model includes the maintenance (or decay) terms $a_{0} x_{0}$ and $a_{1} x_{1}$, where $a_{0}$ and $a_{1}$ are positive parameters. The functions $\mu_{0}(.,$.$) and \mu_{1}($.$) are the$ specific growth rate of the bacteria.

The terms $\mu_{0}\left(s_{0}, s_{1}\right) x_{0}$ and $\mu_{1}\left(s_{1}\right) x_{1}$ in the first and third equations represent the consumption of substrates $s_{0}$ and $s_{1}$ by the biomasses $x_{0}$ and $x_{1}$, respectively. These terms in the second and fourth equations represent the growth of the biomassess $x_{0}$ and $x_{1}$, respectively. The variables have been rescaled such that all the constant parameters were fixed to 1 , see [5] for the details.

We assume that the functions $\mu_{0}(.,$.$) and \mu_{1}($.$) satisfy:$

H1 For all $s_{0}>0$ and $s_{1} \geq 0, \mu_{0}\left(s_{0}, s_{1}\right)>0, \mu_{0}\left(0, s_{1}\right)=0$ and $\sup _{s_{0} \geqslant 0} \mu_{0}\left(s_{0}, s_{1}\right)<+\infty$.

H2 For all $s_{1}>0, \mu_{1}\left(S_{1}\right)>0, \mu_{1}(0)=0$ and $m_{1}:=\sup _{s_{1} \geqslant 0} \mu_{1}\left(s_{1}\right)<+\infty$.

H3 For all $s_{0}>0$ and $s_{1}>0, \frac{\partial \mu_{0}}{\partial s_{0}}\left(s_{0}, s_{1}\right)>0$ and $\frac{\partial \mu_{0}}{\partial s_{1}}\left(s_{0}, s_{1}\right)<0$.

H4 For all $s_{1}>0, \frac{d \mu_{1}}{d s_{1}}\left(s_{1}\right)>0$.

For $s_{1}$ fixed, we denote:

$$
m_{0}\left(s_{1}\right)=\sup _{s_{0} \geqslant 0} \mu_{0}\left(s_{0}, s_{1}\right)
$$

We assume that:

H5 For all $s_{1}>0, \frac{d m_{0}}{d s_{1}}<0$.

Hypothesis $\mathbf{H 1}$ means that no growth can take place for species $x_{0}$ without the substrate $s_{0}$. Hypothesis $\mathbf{H 2}$ means that the intermediate product $s_{1}$ is necessary for the growth of species $x_{1}$. Hypothesis $\mathbf{H 3}$ means that the growth rate of species $x_{0}$ increases with the substrate $s_{0}$ but it is self-inhibited by the intermediate product $s_{1}$. Hypothesis $\mathbf{H 4}$ means that the growth of species $x_{1}$ increases with intermediate product $s_{1}$ produced by species $x_{0}$. Note that this defines a syntrophic relationship between the two species. Hypothesis $\mathbf{H} 5$ means that the maximal growth rate of species $x_{0}$ decreases with the substrate $s_{1}$.

We first state the following result:

Proposition 2.1. For every non-negative initial condition, the solution of (2.1) has non-negative components and is positively bounded and thus is defined for every positive $t$.

The proof is given in the Appendix A. 


\section{Steady state AnAlysis}

A steady state of (2.1) is a solution of the following nonlinear algebraic system obtained by setting the right-hand sides of (2.1) equal to zero:

$$
\begin{array}{r}
D\left(s_{0}^{i n}-s_{0}\right)-\mu_{0}\left(s_{0}, s_{1}\right) x_{0}=0, \\
-D x_{0}+\mu_{0}\left(s_{0}, s_{1}\right) x_{0}-a_{0} x_{0}=0, \\
D\left(s_{1}^{i n}-s_{1}\right)+\mu_{0}\left(s_{0}, s_{1}\right) x_{0}-\mu_{1}\left(s_{1}\right) x_{1}=0, \\
-D x_{1}+\mu_{1}\left(s_{1}\right) x_{1}-a_{1} x_{1}=0,
\end{array}
$$

Since all state variables are concentrations, steady state $E=\left(s_{0}, x_{0}, s_{1}, x_{1}\right)$ exists if and only if all its components are non-negative. From equation (3.2) we deduce that:

$$
x_{0}=0 \quad \text { or } \quad \mu_{0}\left(s_{0}, s_{1}\right)=D+a_{0},
$$

and from equation (3.4) we deduce that:

$$
x_{1}=0 \quad \text { or } \quad \mu_{1}\left(s_{1}\right)=D+a_{1} .
$$

We obtain the four equilibria:

SS0: $x_{0}=0, x_{1}=0$, where both species are washed out.

SS1: $x_{0}>0, x_{1}=0$, where species $x_{1}$ is washed out while $x_{0}$ survives.

SS2: $x_{0}>0, x_{1}>0$, where both species survive.

SS3: $x_{0}=0, x_{1}>0$, where species $x_{0}$ is washed out while $x_{1}$ survives.

For the description of the steady states, we need the following notations. Since the function $s_{1} \mapsto \mu_{1}\left(s_{1}\right)$ is increasing, it has an inverse function $y \mapsto M_{1}(y)$, so that, for all $s_{1} \geq 0$ and $y \in\left[0, m_{1}[\right.$

$$
s_{1}=M_{1}(y) \Longleftrightarrow y=\mu_{1}\left(s_{1}\right) .
$$

Let $s_{1}$ be fixed. Since the function $s_{0} \mapsto \mu_{0}\left(s_{0}, s_{1}\right)$ is increasing, it has an inverse function $y \mapsto M_{0}\left(y, s_{1}\right)$, so that, for all $s_{0}, s_{1} \geq 0$, and $y \in\left[0, m_{0}\left(s_{1}\right)[\right.$

$$
s_{0}=M_{0}\left(y, s_{1}\right) \Longleftrightarrow y=\mu_{0}\left(s_{0}, s_{1}\right) .
$$

Then, we have the following result.

Proposition 3.1. Using assumptions $\mathbf{H 1 - H 4}$, we have:

- For all $y \in\left[0, m_{0}\left(s_{1}\right)\left[\right.\right.$ and $s_{1} \geqslant 0, \frac{\partial M_{0}}{\partial y}\left(y, s_{1}\right)>0$ and $\frac{\partial M_{0}}{\partial s_{1}}\left(y, s_{1}\right)>0$.

- For all $y \in\left[0, m_{1}\left[, \frac{d M_{1}}{d y}(y)>0\right.\right.$.

The proof is given in the Appendix A. Thus, we can prove the following proposition:

Proposition 3.2. Assume that assumptions $\mathbf{H 1 - H 4}$ hold. Then, (2.1) has at most four steady states:

- $S S 0=\left(s_{0}^{i n}, 0, s_{1}^{i n}, 0\right)$. It always exists.

- $S S 1=\left(s_{01}, x_{01}, s_{11}, 0\right)$, where $s_{01}$ is the solution of the equation: $\mu_{0}\left(s_{01},\left(s_{0}^{\text {in }}+s_{1}^{\text {in }}\right)-s_{01}\right)=D+a_{0}$. $x_{01}=\frac{D}{D+a_{0}}\left(s_{0}^{i n}-s_{01}\right)$ and $s_{11}=\left(s_{0}^{i n}+s_{1}^{i n}\right)-s_{01}$.

It exists if and only if $s_{0}^{\text {in }}>M_{0}\left(D+a_{0}, s_{1}^{\text {in }}\right)$. 
- $S S 2=\left(s_{02}, x_{02}, s_{12}, x_{12}\right)$, where $s_{02}=M_{0}\left(D+a_{0}, M_{1}\left(D+a_{1}\right)\right), x_{02}=\frac{D}{D+a_{0}}\left(s_{0}^{i n}-s_{02}\right), s_{12}=M_{1}$ $\left(D+a_{1}\right)$ and $x_{12}=\frac{D}{D+a_{1}}\left(\left(s_{0}^{i n}+s_{1}^{i n}\right)-s_{02}-s_{12}\right)$.

It exists if and only if $s_{0}^{\text {in }}>M_{0}\left(D+a_{0}, M_{1}\left(D+a_{1}\right)\right)$ and $s_{0}^{i n}+s_{1}^{i n}>M_{0}\left(D+a_{0}, M_{1}\left(D+a_{1}\right)\right)+M_{1}$ $\left(D+a_{1}\right)$.

- $S S 3=\left(s_{0}^{i n}, 0, M_{1}\left(D+a_{1}\right), \frac{D}{D+a_{1}}\left(s_{1}^{i n}-M_{1}\left(D+a_{1}\right)\right)\right)$. It exists if and only if $s_{1}^{i n}>M_{1}\left(D+a_{1}\right)$.

The proof is given in the Appendix A. With respect to [5], a new steady state SS3 exists. Notice that, if $s_{1}^{i n}=0$ the condition $\mu_{1}\left(s_{1}^{i n}\right)>a_{1}$ is not satisfied and SS3 does not exist. In the next section, we analyse local stability of the steady states.

\section{Stability ANALYSiS}

The stability of the steady states is given by the sign of the real part of eigenvalues of the Jacobian matrix or by the Routh-Hurwitz criteria (in the case of $S S 2$ ). In the following, we use the abbreviations LES for locally exponentially stable.

Proposition 4.1. Assume that assumptions $\mathbf{H 1}-\mathbf{H} 4$ hold. Then, the local stability of steady states of (2.1) is given by:

- $S S 0$ is LES if and only if $s_{1}^{i n}<M_{1}\left(D+a_{1}\right)$ and $s_{0}^{i n}<M_{0}\left(D+a_{0}, s_{1}^{i n}\right)$.

- $S S 1$ is LES if and only if $s_{0}^{\text {in }}+s_{1}^{\text {in }}<M_{0}\left(D+a_{0}, M_{1}\left(D+a_{1}\right)\right)+M_{1}\left(D+a_{1}\right)$.

- $S S 2$ is LES if it exists.

- $S S 3$ is LES if and only if $s_{0}^{i n}<M_{0}\left(D+a_{0}, M_{1}\left(D+a_{1}\right)\right)$.

The proof is given in the Appendix A. The results of Propositions 3.2 and 4.1 are summarized in Table 1 where the functions $F_{i}, i=0,1,2$, are defined by:

$$
\begin{aligned}
& F_{0}(D)=M_{0}\left(D+a_{0}, s_{1}^{i n}\right) \\
& F_{1}(D)=M_{1}\left(D+a_{1}\right)+M_{0}\left(D+a_{0}, M_{1}\left(D+a_{1}\right)\right), \\
& F_{2}(D)=M_{0}\left(D+a_{0}, M_{1}\left(D+a_{1}\right)\right) .
\end{aligned}
$$

The domains of definition of the functions $F_{i}$, for $i=0,1$ and 2, are given in Proposition 4.2. Notice that:

$$
s_{1}^{i n}<M_{1}\left(D+a_{1}\right) \Longleftrightarrow D>\mu_{1}\left(s_{1}^{i n}\right)-a_{1} .
$$

Proposition 4.2. We have:

- $F_{0}$ is defined in $\left[0, D_{0}\left[\right.\right.$, with $D_{0}=m_{0}\left(s_{1}^{\text {in }}\right)-a_{0}$. This interval is not empty if and only if $a_{0}<m_{0}\left(s_{1}^{i n}\right)$.

- $F_{1}$ is defined in $\left[0, D_{1}\left[\right.\right.$, with $D_{1}=\min \left(m_{1}-a_{1}, D_{2}\right)$ with $D_{2}$ is the positive solution of equation

$D+a_{0}=m_{0}\left(M_{1}\left(D+a_{1}\right)\right)$. $\left[0, D_{1}\left[\right.\right.$ is not empty if and only if $a_{1}<m_{1}$ and $a_{0}<m_{0}\left(M_{1}\left(a_{1}\right)\right)$.

- $F_{2}$ is defined in $\left[0, D_{2}\left[, D_{2}\right.\right.$ exists if and only if $a_{0}<m_{0}\left(M_{1}\left(a_{1}\right)\right)$.

The proof is given in the Appendix A.

\section{Operating DiAgRAMS}

The operating diagrams show how the system behaves when we vary the three operating parameters $s_{0}^{i n}, s_{1}^{\text {in }}$ and $D$.

These diagrams are specially useful for the operators, to estimate in particular, for a given a triplet $s_{0}^{i n}, s_{1}^{i n}$ and $D$, the margin of stability they have, with respect to a region of the space where the washing out of at least one biomass is stable. For a planar operating diagram, we must fix one of the three operating parameters $D, s_{0}^{i n}$ or $s_{1}^{i n}$. In Section 5.1, we fix $s_{1}^{i n}$ and we determine the operating diagrams in the plane $\left(s_{0}^{i n}, D\right)$ and, in Section 5.2 , we give the operating diagrams in the plane $\left(s_{1}^{i n}, D\right)$ with $s_{0}^{i n}$ fixed. 
TABLE 1. Existence and local stability of steady states.

\begin{tabular}{lll}
\hline Steady state & Existence condition & Stability condition \\
\hline SS0 & Always exists & $s_{0}^{i n}<F_{0}(D)$ and $D>\mu_{1}\left(s_{1}^{i n}\right)-a_{1}$ \\
SS1 & $s_{0}^{i n}>F_{0}(D)$ & $s_{0}^{i n}+s_{1}^{i n}<F_{1}(D)$ \\
SS2 & $s_{0}^{i n}+s_{1}^{i n}>F_{1}(D)$ and $s_{0}^{i n}>F_{2}(D)$ & Stable when it exists \\
SS3 & $\mu_{1}\left(s_{1}^{\text {in }}\right)>a_{1}$ and $D<\mu_{1}\left(s_{1}^{\text {in }}\right)-a_{1}$ & $s_{0}^{\text {in }}<F_{2}(D)$ \\
\hline
\end{tabular}

TABLE 2. The cases $\mu_{1}\left(s_{1}^{\text {in }}\right)<a_{1}$.

\begin{tabular}{ccccc}
\hline Condition & Region & SS0 & SS1 & SS2 \\
\hline$F_{0}(D)<s_{0}^{i n}<F_{1}(D)-s_{1}^{i n}$ & $\left(s_{0}^{i n}, D\right) \in R^{1}$ & $\mathrm{U}$ & $\mathrm{S}$ & \\
$s_{0}^{i n}<F_{0}(D)$ & $\left(s_{0}^{i n}, D\right) \in R^{2}$ & $\mathrm{~S}$ & & \\
$F_{1}(D)-s_{1}^{i n}<s_{0}^{i n}$ & $\left(s_{0}^{i n}, D\right) \in R^{6}$ & $\mathrm{U}$ & $\mathrm{U}$ & $\mathrm{S}$ \\
\hline
\end{tabular}

TABLE 3 . The cases $\mu_{1}\left(s_{1}^{i n}\right)>a_{1}$.

\begin{tabular}{ccccccc}
\hline & Condition & Region & SS0 & SS1 & SS2 & SS3 \\
\hline \multirow{4}{*}{$D>\bar{D}$} & $F_{0}(D)<s_{0}^{i n}<F_{1}(D)-s_{1}^{i n}$ & $\left(s_{0}^{i n}, D\right) \in R^{1}$ & $\mathrm{U}$ & $\mathrm{S}$ & & \\
& $s_{0}^{i n}<F_{0}(D)$ & $\left(s_{0}^{i n}, D\right) \in R^{2}$ & $\mathrm{~S}$ & & & \\
& $F_{1}(D)-s_{1}^{i n}<s_{0}^{i n}$ & $\left(s_{0}^{i n}, D\right) \in R^{6}$ & $\mathrm{U}$ & $\mathrm{U}$ & $\mathrm{S}$ & \\
\hline \multirow{2}{*}{$D<\bar{D}$} & $s_{0}^{i n}<F_{2}(D)$ & $\left(s_{0}^{i n}, D\right) \in R^{3}$ & $\mathrm{U}$ & & & $\mathrm{S}$ \\
& $F_{2}(D)<s_{0}^{i n}<F_{0}(D)$ & $\left(s_{0}^{i n}, D\right) \in R^{4}$ & $\mathrm{U}$ & & $\mathrm{S}$ & $\mathrm{U}$ \\
& $s_{0}^{i n}>F_{0}(D)$ & $\left(s_{0}^{i n}, D\right) \in R^{5}$ & $\mathrm{U}$ & $\mathrm{U}$ & $\mathrm{S}$ & $\mathrm{U}$ \\
\hline
\end{tabular}

\subsection{Operating diagram with respect to $\left(s_{0}^{i n}, D\right)$ and $s_{1}^{i n}$ fixed}

In a first step, we fix $s_{1}^{i n}$ and we illustrate the equilibria existence and stability domains in the plane $\left(s_{0}^{i n}, D\right)$. Let $F_{0}(D), F_{1}(D)$ and $F_{2}(D)$ be the functions defined by (4.1). We define the curve $\gamma_{0}$ of equation $s_{0}^{i n}=$ $F_{0}(D)$, the curve $\gamma_{1}$ of equation $s_{0}^{\text {in }}=F_{1}(D)-s_{1}^{\text {in }}$ and the curve $\gamma_{2}$ of equation $s_{0}^{i n}=F_{2}(D)$. We denote $\bar{D}=\mu_{1}\left(s_{1}^{i n}\right)-a_{1}$, see Table 1 .

These curves with the line $D=\bar{D}$ separate the operating plane $\left(s_{0}^{i n}, D\right)$ in at most six regions as shown in Figure 3, labelled $R^{1}, \ldots, R^{6}$.

The results of Proposition 4.1 are summarized in the next theorem which shows the existence and local stability of the steady states SS0, ., SS3 in the regions $R^{1}, \cdots, R^{6}$ of the operating diagram, for a given $s_{1}^{i n}$. The regions $R^{i}, i=1, \ldots, 6$ of operating diagram are colored by four different colors. Each color corresponds to one and only one stable steady-state: in the region $R^{4}, R^{5}$ and $R^{6}$, SS2 exists and is stable. In $R^{5}$, all the other steady states exist but are unstable. In the region $R^{4}$, (respectively $R^{6}$ ), the steady-state SS1 (respectively SS3) does not exist and the other steady-states exist. Therefore these regions are all colored in the same yellow color. Similarly the region $R^{2}$ (in green) is the stability region of the washout steady-state SS0, the region $R^{1}$ (in blue) is the stability region of steady-state SS1 and $R^{3}$ (in purple) is the stability region of steady state SS3. It is useful to state the next properties on the functions $F_{i}, i=0,1,2$.

Lemma 5.1. We have

- If $\mu_{1}\left(s_{1}^{i n}\right)<a_{1}$ then $F_{0}(D)<F_{1}(D)-s_{1}^{i n}$.

- If $\mu_{1}\left(s_{1}^{i n}\right)>a_{1}$ and $D>\mu_{1}\left(s_{1}^{i n}\right)-a_{1}$ then $F_{0}(D)<F_{1}(D)-s_{1}^{\text {in }}$.

- If $\mu_{1}\left(s_{1}^{i n}\right)>a_{1}$ and $D<\mu_{1}\left(s_{1}^{i n}\right)-a_{1}$ then $F_{2}(D)<F_{0}(D)$. 
TABLE 4. Existence and local stability of steady states, according to $s_{1}^{i n}$.

\begin{tabular}{lll}
\hline Steady state & Existence condition & Stability condition \\
\hline SS0 & Always exists & $s_{1}^{i n}>F_{3}(D)$ and $s_{1}^{i n}<F_{1}(D)-F_{2}(D)$ \\
SS1 & $\bar{D}_{3}>0$ and $s_{1}^{i n}<F_{3}(D)$ & $s_{1}^{i n}<F_{1}(D)-s_{0}^{i n}$ \\
SS2 & $s_{1}^{\text {in }}>F_{1}(D)-s_{0}^{i n}, \bar{D}_{1}>0$ and $D<\bar{D}_{1}$ & Stable when it exists \\
SS3 & $s_{1}^{i n}>F_{1}(D)-F_{2}(D)$ & $D>\bar{D}_{1}$ \\
\hline
\end{tabular}

TABLE 5. The cases $\overline{D_{1}}<0, \bar{D}_{3}<0$ and $0<D<\bar{D}_{2} e e e$.

\begin{tabular}{cccc}
\hline Condition & Region & SS0 & SS3 \\
\hline$s_{1}^{\text {in }}<F_{1}(D)-F_{2}(D)$ & $\left(s_{1}^{\text {in }}, D\right) \in R^{2}$ & $\mathrm{~S}$ & \\
$F_{1}(D)-F_{2}(D)<s_{1}^{\text {in }}$ & $\left(s_{1}^{\text {in }}, D\right) \in R^{3}$ & $\mathrm{U}$ & $\mathrm{S}$ \\
\hline
\end{tabular}

TABLE 6 . The cases $\bar{D}_{1}<0, \bar{D}_{3}>0$ and $0<D<\bar{D}_{2}$.

\begin{tabular}{ccccc}
\hline Condition & Region & SS0 & SS1 & SS3 \\
\hline$s_{1}^{\text {in }}<F_{3}(D)$ & $\left(s_{1}^{\text {in }}, D\right) \in R_{1}^{1}$ & $\mathrm{U}$ & $\mathrm{S}$ & \\
$F_{3}(D)<s_{1}^{\text {in }}<F_{1}(D)-F_{2}(D)$ & $\left(s_{1}^{\text {in }}, D\right) \in R^{2}$ & $\mathrm{~S}$ & & \\
$F_{1}(D)-F_{2}(D)<s_{1}^{\text {in }}$ & $\left(s_{1}^{\text {in }}, D\right) \in R^{3}$ & $\mathrm{U}$ & & $\mathrm{S}$ \\
\hline
\end{tabular}

TABLE 7 . The cases $\overline{D_{1}}>0, \bar{D}_{3}>0$ and $0<D<\overline{D_{2}}$.

\begin{tabular}{|c|c|c|c|c|c|c|}
\hline & Conditions & Region & SS0 & SS1 & $\mathrm{SS} 2$ & SS3 \\
\hline $\bar{D}_{1}<D$ & $\begin{array}{c}s_{1}^{i n}<F_{3}(D) \\
F_{3}(D)<s_{1}^{i n}<F_{1}(D)-F_{2}(D) \\
F_{1}(D)-F_{2}(D)<s_{1}^{i n}\end{array}$ & $\begin{array}{l}\left(s_{1}^{i n}, D\right) \in R_{1}^{1} \\
\left(s_{1}^{\text {in }}, D\right) \in R^{2} \\
\left(s_{1}^{\text {in }}, D\right) \in R^{3}\end{array}$ & $\begin{array}{l}\mathrm{U} \\
\mathrm{S} \\
\mathrm{U}\end{array}$ & $\mathrm{S}$ & & $\mathrm{S}$ \\
\hline$D<\bar{D}_{1}$ & $\begin{aligned} F_{3}(D) & <s_{1}^{i n} \\
F_{1}(D)-F_{2}(D) & <s_{1}^{i n}<F_{3}(D) \\
F_{1}(D)-s_{0}^{i n} & <s_{1}^{i n}<F_{1}(D)-F_{2}(D) \\
s_{1}^{i n} & <F_{1}(D)-s_{0}^{i n}\end{aligned}$ & $\begin{array}{l}\left(s_{1}^{i n}, D\right) \in R^{4} \\
\left(s_{1}^{\text {in }}, D\right) \in R^{5} \\
\left(s_{1}^{\text {in }}, D\right) \in R^{6} \\
\left(s_{1}^{\text {in }}, D\right) \in R_{2}^{1}\end{array}$ & $\begin{array}{l}\mathrm{U} \\
\mathrm{U} \\
\mathrm{U} \\
\mathrm{U}\end{array}$ & $\begin{array}{l}\mathrm{U} \\
\mathrm{U} \\
\mathrm{S}\end{array}$ & $\begin{array}{l}\text { S } \\
\text { S } \\
\text { S }\end{array}$ & $\begin{array}{l}\mathrm{U} \\
\mathrm{U}\end{array}$ \\
\hline
\end{tabular}

For a detailed proof, see the Appendix A. We can now state the following result:

Theorem 5.2. The existence and stability properties of the system (2.1), in the plane $\left(s_{0}^{i n}, D\right)$, are summarized in the following tables:

The letter $S$ (resp. U) means that the corresponding equilibrium is LES (resp. unstable). The absence of letter means that the equilibrium does not exist.

The proof is given in the Appendix A.

These results are essentially the same as those presented in Table 1 . Notice that Table 2 is identical to the Table 2 of [5], it corresponds to the case where the concentration $s_{1}^{i n}$ is small or equal to zero. Table 3 emerges due to the presence of $s_{1}^{i n}$ : three regions - where SS3 exists - appear. Moreover, in the regions $R^{i}, i=1, \ldots, 6$, there is only one stable steady state and all other equilibria are unstable or not even exist. 
TABLE 8. Nominal parameters values for a syntrophic model of degradation of fatty acids and hydrogen by the acetogenic bacteria and methanogenic hydrogenetrophic bacteria, respectively.

\begin{tabular}{lll}
\hline Parameters & Units & Nominal values \\
\hline$m_{0}$ & $\mathrm{~d}^{-1}$ & 0.52 \\
$K_{0}$ & $\mathrm{~kg} \mathrm{COD} / \mathrm{m}^{3}$ & 0.124 \\
$m_{1}$ & $\mathrm{~d}^{-1}$ & 2.10 \\
$K_{1}$ & $\mathrm{~kg} \mathrm{COD} / \mathrm{m}^{3}$ & 0.25 \\
$K_{i}$ & $\mathrm{~kg} \mathrm{COD} / \mathrm{m}^{3}$ & 0.035 \\
$a_{0}$ & $\mathrm{~d}^{-1}$ & 0.02 \\
$a_{1}$ & $\mathrm{~d}^{-1}$ & 0.02 \\
\hline
\end{tabular}
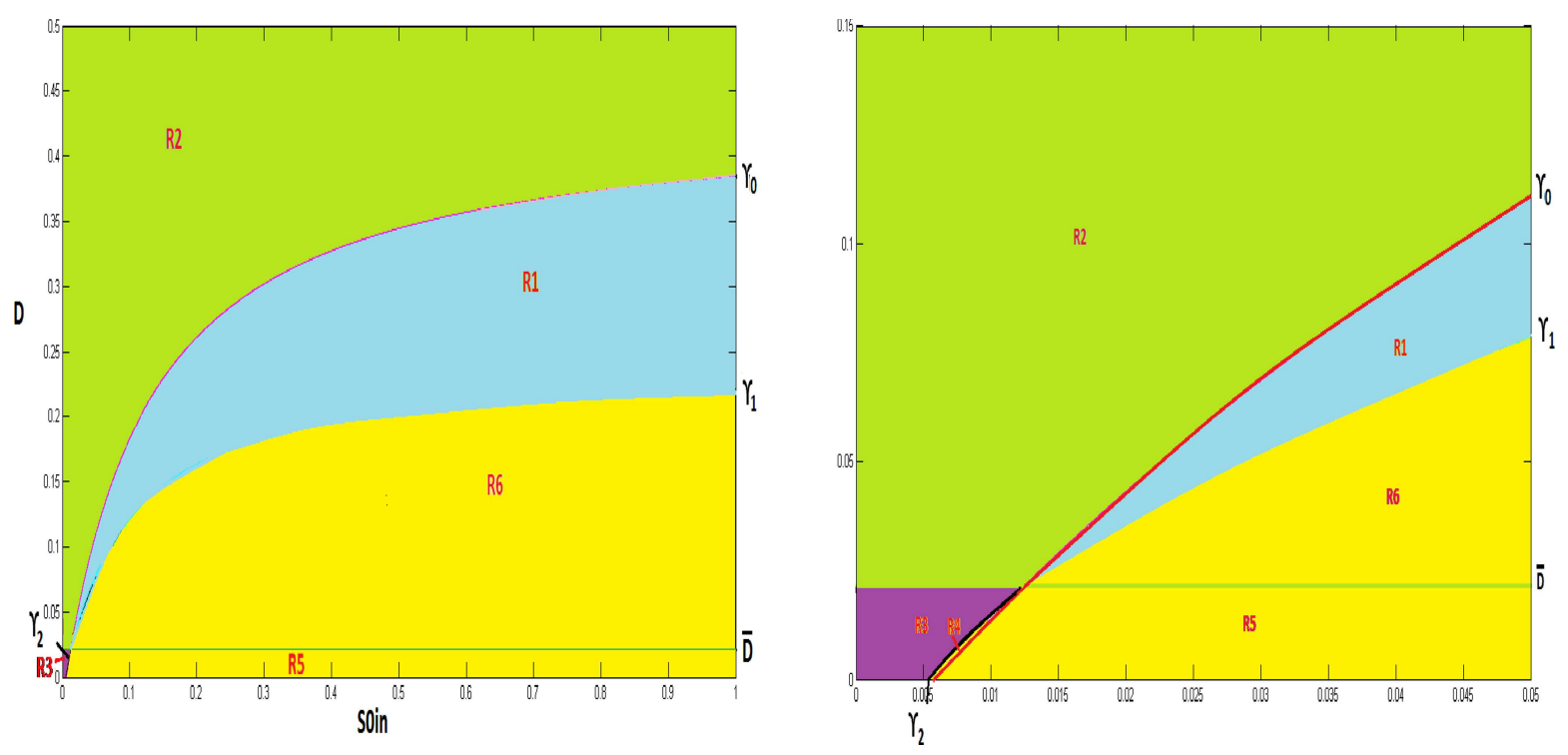

Figure 2. Operating diagram of the model (2.1) for $s_{1}^{\text {in }}=0.005 \mathrm{KgCODm}^{-3}$ and $\bar{D}=$ $0.021 d^{-1}$. The region $R^{2}$ (in green) is the stability region of the washout steady-state SS0, $R^{1}$ (in blue) is the stability region of steady-state SS1 $, R^{6}, R^{5}$ and $R^{4}$ (in yellow) are the stability region of steady-state SS2 and the region $R^{3}$ (in purple) is the stability region of steady-state SS3. (the figure at right is a zoom of the bottom of the figure at left)

\subsection{Operating diagram with respect to $\left(s_{1}^{i n}, D\right)$ and $s_{0}^{i n}$ fixed}

Now, let $s_{0}^{\text {in }}$ be fixed. Since the function $s_{1} \mapsto \mu_{0}\left(s_{0}, s_{1}\right)$ is decreasing, it has a decreasing inverse function $z \mapsto M_{2}\left(s_{0}, z\right)$, so that, for all $s_{0}, s_{1} \geq 0$, and $z \in\left[0, \sup \mu_{0}\left(s_{0}, \cdot\right)[\right.$

$$
s_{1}=M_{2}\left(s_{0}, z\right) \Longleftrightarrow z=\mu_{0}\left(s_{0}, s_{1}\right) .
$$

We define the function:

$$
F_{3}(D)=M_{2}\left(s_{0}^{i n}, D+a_{0}\right) .
$$

Let $\bar{D}_{1}$, if it exists, be the largest solution of $F_{2}(D)=s_{0}^{\text {in }}$, and $\bar{D}_{2}=\min \left(m_{1}-a_{1}, D_{2}\right)$, such that $F_{1}$ is defined in $\left[0, \bar{D}_{2}\left[\right.\right.$. Let $\bar{D}_{3}$ the solution of $F_{3}(D)=0$. Since $F_{3}$ is decreasing, then $\bar{D}_{3}<0$ implies that $F_{3}(D)<0$. 


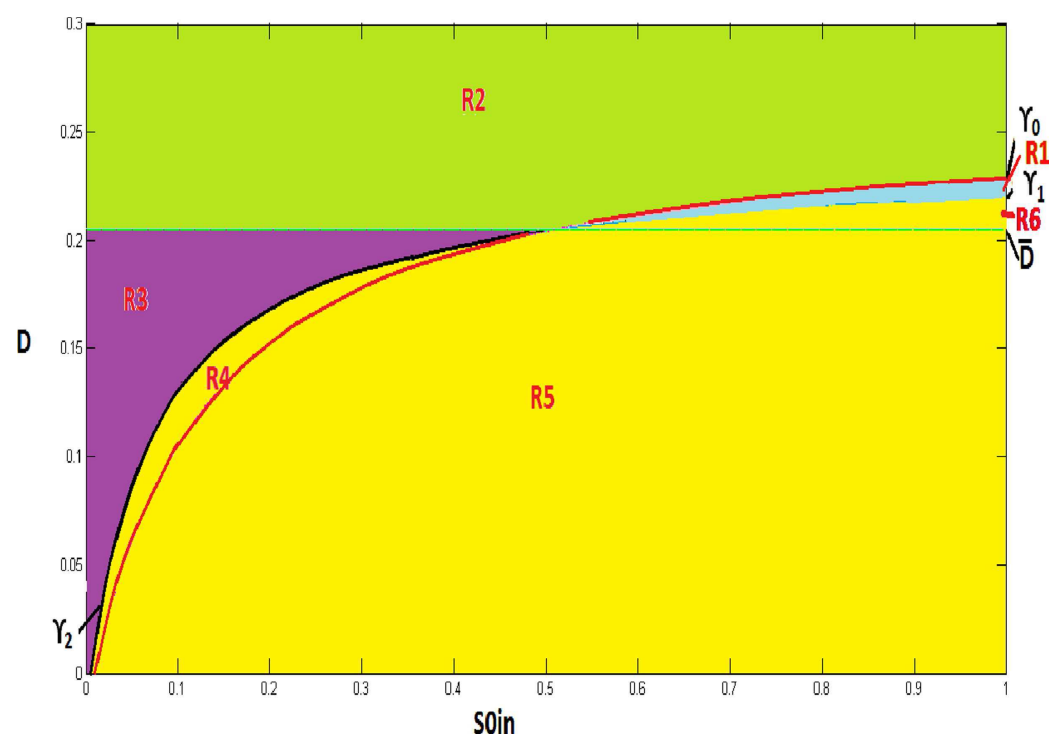

Figure 3. Operating diagram of the model (2.1) for $s_{1}^{i n}=0.03 \mathrm{KgCODm}^{-3}$ and $\bar{D}=$ $0.205 d^{-1}$.

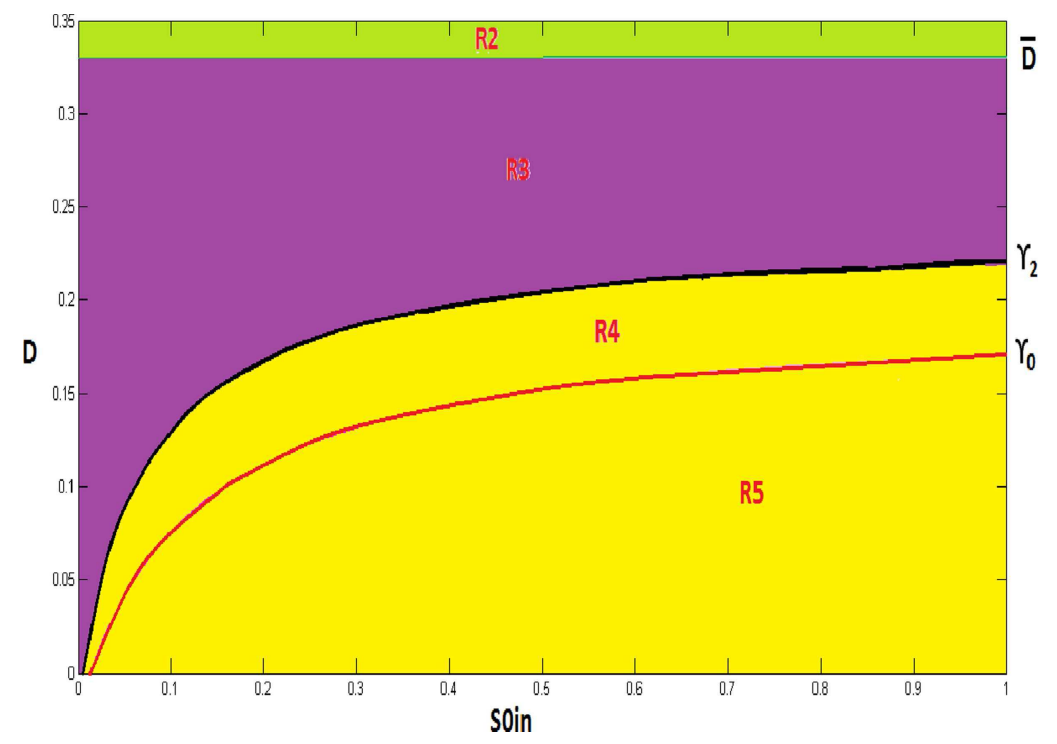

FiguRE 4. Operating diagram of the model (2.1) for $s_{1}^{i n}=0.05 \mathrm{KgCODm}^{-3}$ and $\bar{D}=0.33 d^{-1}$.

To illustrate the regions of existence and stability of the steady states in the plane $\left(s_{1}^{i n}, D\right)$, we first express the conditions of Table 1 according to $s_{1}^{i n}$, which gives the following table:

It is useful to state the next properties on the functions $F_{i}, i=1,2,3$.

Lemma 5.3. We assume that $\overline{D_{2}}>0$. Then, we have

- If $D>\bar{D}_{1}$ then $F_{3}(D)<F_{1}(D)-F_{2}(D)<F_{1}(D)-s_{0}^{\text {in }}$.

- If $D<\bar{D}_{1}$ and $\bar{D}_{1}>0$ then $F_{1}(D)-s_{0}^{\text {in }}<F_{1}(D)-F_{2}(D)<F_{3}(D)$. Moreover, the three curves of functions $F_{1}-F_{2}, F_{1}-s_{0}^{\text {in }}$ and $F_{3}$ intersect at $D=\bar{D}_{1}$ satisfying $\bar{D}_{3}>\bar{D}_{1}$. 


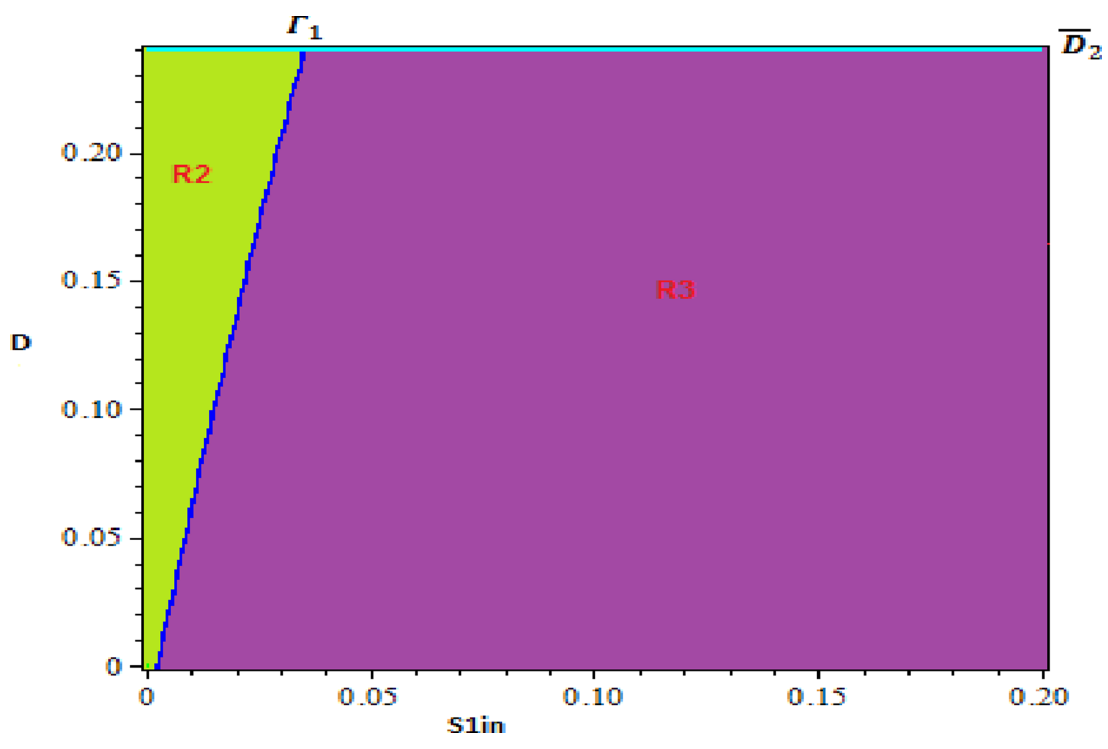

Figure 5. Operating diagram of the model (2.1) for $s_{0}^{i n}=0.005 \mathrm{KgCODm}^{-3}$.

For a detailed proof, see the Appendix A.

The regions $R^{i}, i=1, \ldots, 6$ appear in the plane $\left(s_{1}^{i n}, D\right)$ as the regions delimited by the following curves : $\Gamma_{0}$ is the curve of the function $s_{1}^{i n}=F_{1}(D)-s_{0}^{i n}, \Gamma_{1}$ is the curve of the function $s_{1}^{i n}=F_{1}(D)-F_{2}(D)$ and $\Gamma_{2}$ is the curve of the function $s_{1}^{i n}=F_{3}(D)$. These curves with the line $D=\bar{D}_{1}$ separate the operating plane $\left(s_{1}^{i n}, D\right)$ in at most six regions as shown in Figure 6. We notice that the region $R^{1}$ is divided into two subregions defined as follows $R^{1}=R_{1}^{1} \cup R_{2}^{1}$.

We can now state the following result:

Theorem 5.4. The existence and stability properties of the system (2.1), in the plane $\left(s_{1}^{i n}, D\right)$, are given in the following tables:

The proof is given in the Appendix A.

\section{Simulations}

The stability regions of steady states are given by the operating diagram in the plane $\left(s_{0}^{i n}, D\right)$ in Figures $2-4$, for different values of $s_{1}^{i n}$. For the simulations, we use the following growth functions:

$$
\mu_{0}\left(s_{0}, s_{1}\right)=\frac{m_{0} s_{0}}{K_{0}+s_{0}} \frac{1}{1+s_{1} / K_{i}}, \quad \mu_{1}\left(s_{1}\right)=\frac{m_{1} s_{1}}{K_{1}+s_{1}}
$$

For the operating diagrams in Figure 2, 3 and 4, we use the parameters of Table 3 of [5] and obtained from Table 1 of [11], see Table 8 .

The inverse functions $M_{1}($.$) and M_{0}\left(., s_{1}\right)$ of the functions $\mu_{1}($.$) and \mu_{0}\left(., s_{1}\right)$ can be calculated explicitly: we have

$$
\begin{gathered}
y \in\left[0, m_{1}\left[\mapsto M_{1}(y)=\frac{K_{1} y}{m_{1}-y},\right.\right. \\
y \in\left[0, \frac{m_{0}}{1+s_{1} / K_{i}}\left[\mapsto M_{0}\left(y, s_{1}\right)=\frac{K_{0} y}{\frac{m_{0}}{1+s_{1} / K_{i}}-y} .\right.\right.
\end{gathered}
$$




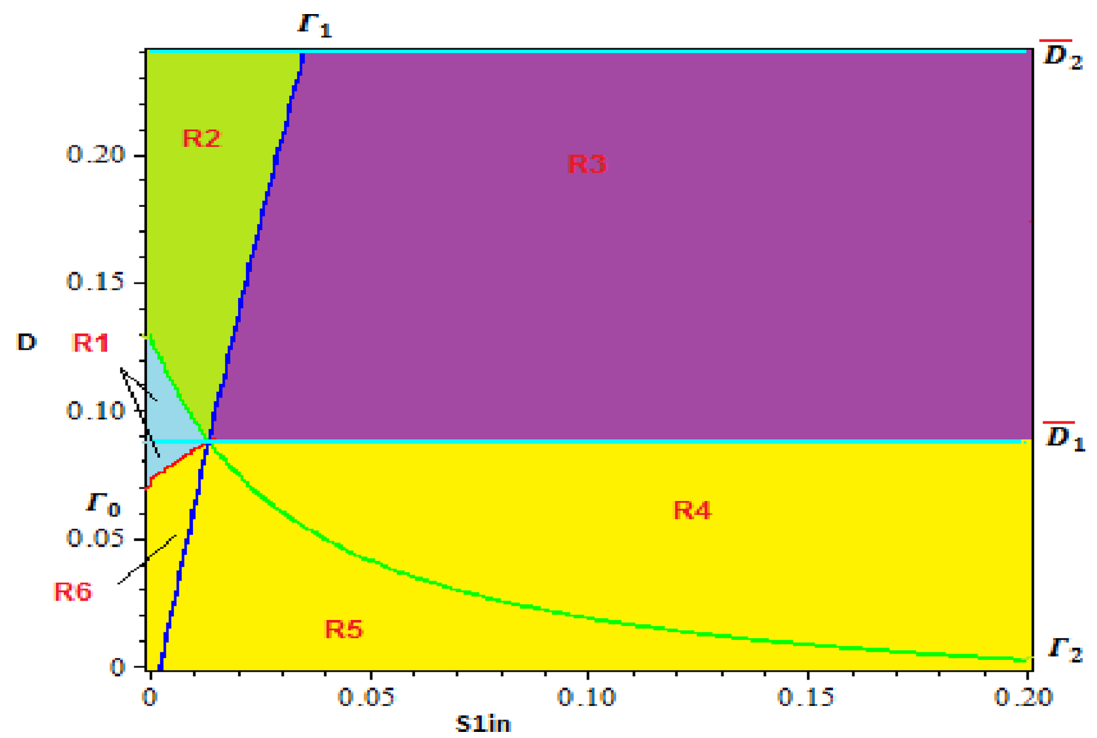

Figure 6. Operating diagram of the model (2.1) for $s_{0}^{i n}=0.05 \mathrm{KgCODm}^{-3}$.

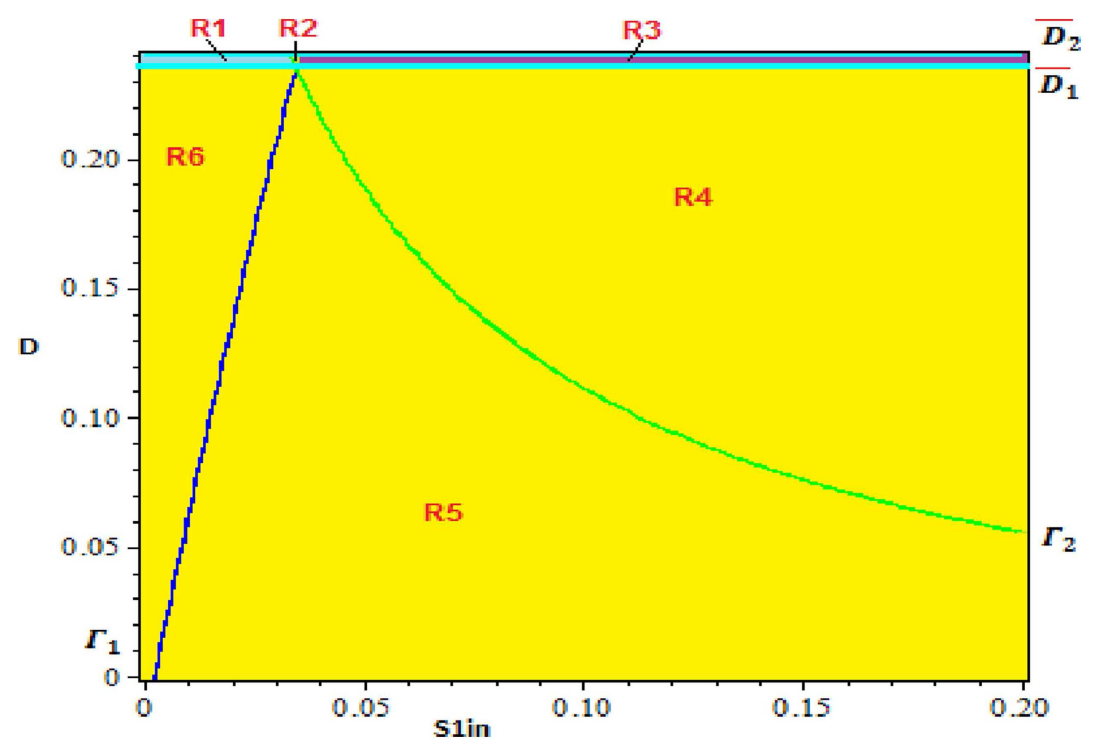

FiguRe 7. Operating diagram of the model (2.1) for $s_{0}^{i n}=5 \mathrm{KgCODm}^{-3}$.

The functions $F_{0}(D), F_{1}(D)$ and $F_{2}(D)$ are given explicitly by

$$
\begin{aligned}
F_{0}(D) & =\frac{K_{0}\left(D+a_{0}\right)\left(1+\frac{s_{1}^{i n}}{K_{i}}\right)}{m_{0}-\left(D+a_{0}\right)\left(1+\frac{s_{1}^{i n}}{K_{i}}\right)}, \\
F_{1}(D) & =\frac{K_{1}\left(D+a_{1}\right)}{m_{1}-\left(D+a_{1}\right)}+\frac{K_{0}\left(D+a_{0}\right)\left(1+\frac{M_{1}\left(D+a_{1}\right)}{K_{i}}\right)}{m_{0}-\left(D+a_{0}\right)\left(1+\frac{M_{1}\left(D+a_{1}\right)}{K_{i}}\right)}, \\
F_{2}(D) & =\frac{K_{0}\left(D+a_{0}\right)\left(1+\frac{M_{1}\left(D+a_{1}\right)}{K_{i}}\right)}{m_{0}-\left(D+a_{0}\right)\left(1+\frac{M_{1}\left(D+a_{1}\right)}{K_{i}}\right)} .
\end{aligned}
$$


$F_{0}$ is defined if $D<\frac{m_{0}-a_{0}\left(1+\frac{s_{1}^{i n}}{K_{i}}\right)}{1+\frac{s_{1}^{i n}}{K_{i}}}$ and $\frac{\left(m_{0}-a_{0}\right) K_{i}}{a_{0}} \geq s_{1}^{i n}$.

$F_{1}$ is defined if $D<m_{1}-a_{1}$ and $\left(K_{i}-K_{1}\right) D^{2}+\left(\left(K_{i}-K_{1}\right)\left(a_{0}+a_{1}\right)-K_{i}\left(m_{1}+m_{0}\right)\right) D+\left(\left(m_{0}-a_{0}\right) K_{i}\left(m_{1}-\right.\right.$ $\left.\left.a_{1}\right)-a_{0} a_{1} K_{1}\right)>0$,

$F_{2}$ is defined if $\left(K_{i}-K_{1}\right) D^{2}+\left(\left(K_{i}-K_{1}\right)\left(a_{0}+a_{1}\right)-K_{i}\left(m_{1}+m_{0}\right)\right) D+\left(\left(m_{0}-a_{0}\right) K_{i}\left(m_{1}-a_{1}\right)-a_{0} a_{1} K_{1}\right)>0$,

that is to say if $D \in\left[0, \bar{D}_{2}[\right.$.

Figures 2-4 illustrate the operating diagrams for increasing values of $s_{1}^{i n}$. When $s_{1}^{\text {in }}$ is small, namely $s_{1}^{i n}=$ 0.005, the most important regions are the regions $R^{i}, i=1,2,6$, (see Fig. 2). These regions correspond to those obtained in the case $s_{1}^{i n}=0$, see (Fig. 1 of [5]). Increasing $s_{1}^{i n}$ leads to the emergence of the existence region of equilibrium SS3 $R^{i}, i=3,4,5$ and to the reduction of the region $R^{1}$ and $R^{6}$, (see Figs. 3 and 4). Thus, the input concentration of the second species leads to the emergence of a new region related to the new equilibrium SS3 and to changes in the size of the existence and stability regions of the other equilibria.

Including $s_{1}^{i n}$ in the model changes slightly the operating diagram of [5]. On the first side, when $s_{1}^{i n}$ increases, $\bar{D}$ increases (it may be verified that $\frac{d \bar{D}}{d s_{1}^{2 n}}>0$ ). The stability region of SS2 under the curve $\gamma_{2}$ remains the same $\left(\gamma_{2}\right.$ does not depend to $\left.s_{1}^{i n}\right)$. On the other side, the stability region $R^{3}$ of SS3, which corresponds to the extinction of the first species, increases in size. When the dilution value of $D$ is small and $S_{0 i n}$ large, the coexistence steady-state is stable. If $D$ large and $S_{0 i n}$ small, then washout steady-state is stable.

The stability region of steady states are given by the operating diagram in the plane $\left(s_{1}^{\text {in }}, D\right)$, see Figures $5-7$, for different values of $s_{0}^{i n}$. The function $F_{3}$ is given by:

$$
F_{3}(D)=\frac{m_{0} K_{i} s_{0}^{i n}}{\left(D+a_{0}\right)\left(K_{0}+s_{0}^{i n}\right)}-K_{i}
$$

$F_{3}$ is defined if $\frac{m_{0} K_{i} s_{0}^{i n}-a_{0} K_{i}\left(K_{0}+s_{0}^{i n}\right)}{K_{i}\left(K_{0}+s_{0}^{i n}\right)} \geq D$ and $s_{0}^{i n} \geq \frac{a_{0} K_{0}}{m_{0}-a_{0}}$.

When $s_{0}^{\text {in }}$ increases, $\bar{D}_{1}$ increases and new regions $R^{4}, \ldots, R^{6}$ appear under the line $D=\bar{D}_{1}$ and $\Gamma_{0}$. This regions correspond to the stability region of the coexistence steady state $S S 2 . \bar{D}_{2}=0.24 d^{-1}$. It does not depend on the values of $s_{0}^{i n}$. When $\bar{D}_{1}$ increases the regions $R^{1}, R^{2}$ and $R^{3}$ become very small, see Figure 7 .

\section{DisCussion}

We have considered a model of an ecosystem involving two bacteria in a chemostat where there are two resources in the input. More precisely, we have proposed a mathematical model involving a syntrophic relationship of two bacteria. For one of the populations, one resource is needed for its growth and the other is inhibitory for the other population growth. One of the populations produces as a by-product the resource that is inhibitory to itself but needed for growth by the other population.

Extending the model studied in [5] by considering that there may have some $s_{1 i n}$ in the influent and using a more general class of kinetics functions, we show that the qualitative behavior of the system can be significantly modified. We have highlighted the existence of a new equilibrium point corresponding to the washout of the first species and the existence of the second.

By using the operating diagram, we can show how the system behaves when we vary the three operating parameters $s_{0}^{i n}, s_{1}^{i n}$ and $D$ while varying the two others in given ranges. To plot the operating diagrams in the plan, we must fixe one of the three operating parameters $s_{0}^{i n}, s_{1}^{i n}$ or $D$. We determine first the operating diagrams in the plane $\left(s_{0}^{i n}, D\right)$, for fixed values of $s_{1}^{i n}$. Then, we fix $s_{0}^{\text {in }}$ and the stability regions are described in the plane $\left(s_{1}^{i n}, D\right)$. We can also fix the dilution rate $D$, if needed, and give the stability regions in the plane $\left(s_{1}^{i n}, s_{0}^{i n}\right)$. For sake of brevity, we do not give this last diagram, in the present work. 
The operating diagrams are divided at most into six regions, colored into four different colors corresponding to the stability regions of the four steady-states. In all cases, we have shown that, whatever the region of space considered, there exists only one locally exponentially stable steady state.

The operating diagrams can be useful to interpret experimental results. The biologists use the results of operating diagrams to know what value of operating parameters to choose for controlling the biogaz (methane or hydrogen) rate product. In particular, $R^{4}, R^{5}$ and $R^{6}$ are the regions of interest for an operator (regions where the coexistence of all species is guaranteed). To optimize the process, one may now couple the informations provided by these diagrams together with plots representing the total amount of biogas produced. Then, two cases may arise: either the operator can act on the input substrate characteristics (for instance in combining several substrate deposits for instance within the framework of codigestion) or he can predict the issue of the process performance given input characteristics.

\section{ApPendix A}

Proof of Proposition 2.1. For all initial condition $s_{0}(0) \geqslant 0$, if it exists one first time $t_{0}>0$ such as $s_{0}\left(t_{0}\right)=0$, then we have $\dot{s_{0}}\left(t_{0}\right)=D s_{0}^{i n}>0$. Therefore $s_{0}(t)>0$ for all $t>t_{0}$. Since $s_{0}(t) \geqslant 0$ for all $t \in\left[0, t_{0}\right]$, then $s_{0}(t) \geqslant 0$ for all $t \geqslant 0$.

On the other hand, for all initial conditions $x_{i}(t) \geqslant 0$ for $i=1,2$, if it exists one first time $t_{0}>0$ such as $x_{i}\left(t_{0}\right)=0$, then we have $\dot{x}_{i}\left(t_{0}\right)=0$, then $x_{i}(t)$ are null from this time $t_{0}$, then $x_{i}(t) \geqslant 0$ for all $t \geqslant 0$.

Finally, for all initial condition $s_{1}(0) \geqslant 0$, if it exists one first time $t_{0}>0$ such as $s_{1}\left(t_{0}\right)=0$, we obtained $\dot{s}_{1}\left(t_{0}\right)=\mu_{0}\left(s_{0}, 0\right) x_{0}+D s_{1}^{i n}>0$. Therefore $s_{1}(t) \geqslant 0$ for all $t>t_{0}$. Since $s_{1}(t) \geqslant 0$ for all $t \in\left[0, t_{0}\right]$, then $s_{1}(t) \geqslant 0$ for all $t \geqslant 0$.

This proves the positivity of solutions of (2.1).

To demonstrate that all solutions of (1) are bounded, we set $z=2 s_{0}+x_{0}+s_{1}+x_{1}$

then

$$
\dot{z}=D\left(2 s_{0}^{i n}+s_{1}^{i n}-z\right)-a_{0} x_{0}-a_{1} x_{1} .
$$

We deduce that, $\dot{z} \leqslant D\left(2 s_{0}^{i n}+s_{1}^{\text {in }}-z\right)$. We now set

$$
v=z-2 s_{0}^{i n}-s_{1}^{i n}
$$

then, $\dot{v} \leqslant-D v$. By applying Gronwall Lemma, we obtain $v(t) \leqslant v(0) e^{-D t}$ and consequently

$$
z(t) \leqslant\left(2 s_{0}^{i n}+s_{1}^{i n}\right)+\left(-2 s_{0}^{i n}-s_{1}^{i n}+z(0)\right) e^{-D t}, \text { for all } t \geqslant 0 .
$$

We deduce that

$$
z(t) \leqslant \max \left(z(0), 2 s_{0}^{i n}+s_{1}^{i n}\right) \text { for all } t \geqslant 0 .
$$

Concequently, the solutions of (2.1) are bounded for all $t \geqslant 0$.

Proof of Proposition 3.1. From the equivalence

$$
s_{0}=M_{0}\left(y, s_{1}\right) \Longleftrightarrow y=\mu_{0}\left(s_{0}, s_{1}\right),
$$

we have:

$$
\text { for all } y \in\left[0, m_{0}\left(s_{1}\right)\left[\text { and } s_{1} \geqslant 0, \quad \mu_{0}\left(M_{0}\left(y, s_{1}\right), s_{1}\right)=y\right. \text {. }\right.
$$


Then, if we take the derivative of equation (A.1) according to $y$ and we use H3, we obtain:

$$
\frac{\partial M_{0}}{\partial y}\left(y, s_{1}\right)=\left[\frac{\partial \mu_{0}}{\partial s_{0}}\left(M_{0}\left(y, s_{1}\right), s_{1}\right)\right]^{-1}>0 .
$$

Now, if we take the derivative of equation (A.1) according to $s_{1}$ and we use H3, we obtain:

$$
\frac{\partial M_{0}}{\partial s_{1}}\left(y, s_{1}\right)=-\left[\frac { \partial \mu _ { 0 } } { \partial s _ { 1 } } ( M _ { 0 } ( y , s _ { 1 } ) , s _ { 1 } ] \left[\frac{\partial \mu_{0}}{\partial s_{0}}\left(M_{0}\left(y, s_{1}\right), s_{1}\right]^{-1}>0 .\right.\right.
$$

Finally, from the equivalence $s_{1}=M_{1}(y) \Longleftrightarrow y=\mu_{1}\left(s_{1}\right)$, we have for all $y \in\left[0, m_{1}\left[, \mu_{1}\left(M_{1}(y)\right)=y\right.\right.$. Taking the derivative of this equation according to $y$ and using $\mathbf{H 4}$, we obtain:

$$
\frac{d M_{1}}{d y}(y)=\left[\frac{\partial \mu_{1}}{\partial s_{1}}\left(M_{1}(y)\right)\right]^{-1}>0
$$

Proposition 3.1 is necessary to establish the results of Proposition 3.2.

Proof of Proposition 3.2. A steady state $\left(s_{0}, x_{0}, s_{1}, x_{1}\right)$ of $(2.1)$ is a solution of the set of algebraic equations (3.1)-(3.4).

- For SS0, $x_{0}=0, x_{1}=0$. As a result of (3.1) and (3.3), we deduce that $s_{0}=s_{0}^{\text {in }}$ and $s_{1}=s_{1}^{i n}$.

Then, $S S 0=\left(s_{0}^{i n}, 0, s_{1}^{i n}, 0\right)$. It always exists.

- For SS1, $x_{0} \neq 0, x_{1}=0$. As a consequence of (3.2), we deduce that $\mu_{0}\left(s_{0}, s_{1}\right)=D+a_{0}$. We have

$$
D\left(s_{0}^{i n}-s_{0}\right)=\mu_{0}\left(s_{0}, s_{1}\right) x_{0} \quad \text { and } \quad D\left(s_{1}-s_{1}^{i n}\right)=\mu_{0}\left(s_{0}, s_{1}\right) x_{0} .
$$

Hence, $x_{0}=\frac{D}{D+a_{0}}\left(s_{0}^{i n}-s_{0}\right)$ and $D\left(s_{0}^{i n}-s_{0}\right)=D\left(s_{1}-s_{1}^{i n}\right)$, so that $s_{0}+s_{1}=s_{0}^{i n}+s_{1}^{i n}$. Therefore, $s_{0}$ is a solution of equation

$$
\mu_{0}\left(s_{0}, s_{0}^{i n}+s_{1}^{i n}-s_{0}\right)=D+a_{0}
$$

SS1 exists if and only if this equation has a solution in the interval $\left(0, s_{0}^{i n}+s_{1}^{i n}\right)$.

The function

$$
s_{0} \mapsto \psi\left(s_{0}\right)=\mu_{0}\left(s_{0}, s_{0}^{i n}+s_{1}^{i n}-s_{0}\right)
$$

is strictly increasing since its derivative

$$
\frac{d \psi}{d s_{0}}\left(s_{0}\right)=\frac{\partial \mu_{0}}{\partial s_{0}}\left(s_{0}, s_{1}\right)-\frac{\partial \mu_{0}}{\partial s_{1}}\left(s_{0}, s_{1}\right)
$$

is positive.

Using $\psi(0)=0$ and $\psi\left(s_{0}^{i n}+s_{1}^{i n}\right)=\mu_{0}\left(s_{0}^{i n}+s_{1}^{i n}, 0\right)$ we conclude that equation $\mu_{0}\left(s_{0}, s_{0}^{i n}+s_{1}^{i n}-s_{0}\right)=D+a_{0}$ has a solution in the interval $\left(0, s_{0}^{i n}+s_{1}^{\text {in }}\right)$ if and only if $\psi\left(s_{0}^{i n}+s_{1}^{i n}\right)=\mu_{0}\left(s_{0}^{i n}+s_{1}^{i n}, 0\right)>D+a_{0}$, which means that:

$$
s_{0}^{i n}+s_{1}^{i n}>M_{0}\left(D+a_{0}, 0\right)
$$


Now, SS1 exists if and only if all his components are strictly positive. For that, it's sufficient that $s_{0}<s_{0}^{i n}$ because $s_{0}^{i n}<s_{0}^{i n}+s_{1}^{i n}$. By applying $\psi$ who is strictly increasing and by using $\mu_{0}$, we obtain: $D+a_{0}<$ $\mu_{0}\left(s_{0}^{i n}, s_{1}^{i n}\right)$ which is equivalent to say that:

$$
s_{0}^{\text {in }}>M_{0}\left(D+a_{0}, s_{1}^{\text {in }}\right) .
$$

Since $s_{0}^{i n}<s_{0}^{i n}+s_{1}^{i n}$, using the same arguments, we obtain: $\mu_{0}\left(s_{0}^{i n}, s_{1}^{i n}\right)<\mu_{0}\left(s_{0}^{i n}+s_{1}^{i n}, 0\right)$. So, if

$$
D+a_{0}<\mu_{0}\left(s_{0}^{i n}, s_{1}^{i n}\right),
$$

then, necessarily

$$
D+a_{0}<\mu_{0}\left(s_{0}^{i n}+s_{1}^{i n}, 0\right) .
$$

Therefore, SS1 exists if and only if

$$
s_{0}^{i n}>M_{0}\left(D+a_{0}, s_{1}^{i n}\right) .
$$

Then, $S S 1=\left(s_{01}, x_{01}, s_{11}, 0\right)$, where $s_{01}$ is the solution of the equation: $\mu_{0}\left(s_{01},\left(s_{0}^{i n}+s_{1}^{i n}\right)-s_{01}\right)=D+a_{0}, x_{01}=\frac{D}{D+a_{0}}\left(s_{0}^{i n}-s_{01}\right)$ and $s_{11}=\left(s_{0}^{i n}+s_{1}^{i n}\right)-s_{01}$. It exists if and only if $s_{0}^{\text {in }}>M_{0}\left(D+a_{0}, s_{1}^{i n}\right)$.

- For SS2, $x_{0} \neq 0$ et $x_{1} \neq 0$. As a consequence of (3.2) and (3.4), we deduce that $s_{0}$ and $s_{1}$ are solutions of the set of equations

$$
\mu_{0}\left(s_{0}, s_{1}\right)=D+a_{0}, \quad \mu_{1}\left(s_{1}\right)=D+a_{1} .
$$

Applying $M_{1}$, we obtain $s_{1}=M_{1}\left(D+a_{1}\right)$ and $s_{0}$ is a solution of equation

$$
\mu_{0}\left(s_{0}, M_{1}\left(D+a_{1}\right)\right)=D+a_{0}
$$

Applying $M_{0}$, we obtain $s_{0}=M_{0}\left(D+a_{0}, M_{1}\left(D+a_{1}\right)\right)$. As a result of (3.1) and (3.3)

$$
x_{0}=\frac{D}{D+a_{0}}\left(s_{0}^{i n}-s_{0}\right), \quad x_{1}=\frac{D}{D+a_{1}}\left(s_{0}^{i n}+s_{1}^{i n}-s_{0}-s_{1}\right) .
$$

SS2 exists if and only if $s_{0}^{i n}+s_{1}^{i n}>s_{0}+s_{1}$ and $s_{0}^{i n}>s_{0}$. This means that:

$$
s_{0}^{i n}+s_{1}^{i n}>M_{0}\left(D+a_{0}, M_{1}\left(D+a_{1}\right)\right)+M_{1}\left(D+a_{1}\right),
$$

and

$$
\left.s_{0}^{i n}>M_{0}\left(D+a_{0}, M_{1}\left(D+a_{1}\right)\right)\right) .
$$

Then, $S S 2=\left(s_{02}, x_{02}, s_{12}, x_{12}\right)$, where $s_{02}=M_{0}\left(D+a_{0}, M_{1}\left(D+a_{1}\right)\right), \quad x_{02}=\frac{D}{D+a_{0}}\left(s_{0}^{i n}-s_{02}\right)$, $s_{12}=M_{1}\left(D+a_{1}\right)$ and $x_{12}=\frac{D}{D+a_{1}}\left(\left(s_{0}^{i n}+s_{1}^{i n}\right)-s_{02}-s_{12}\right)$. It exists if and only if $s_{0}^{i n}>M_{0}$ $\left(D+a_{0}, M_{1}\left(D+a_{1}\right)\right)$ and $s_{0}^{i n}+s_{1}^{i n}>M_{0}\left(D+a_{0}, M_{1}\left(D+a_{1}\right)\right)+M_{1}\left(D+a_{1}\right)$.

- For SS3, $x_{0}=0$ et $x_{1} \neq 0$. As a consequence of (3.1) and (3.4), we deduce that $s_{0}=s_{0}^{i n}$ and $s_{1}$ are solution of this equation

$$
\mu_{1}\left(s_{1}\right)=D+a_{1}
$$


Applying $M_{1}$, we obtain

$$
s_{1}=M_{1}\left(D+a_{1}\right)
$$

As a result of (4), we have:

$$
x_{1}=\frac{D}{D+a_{1}}\left(s_{1}^{i n}-M_{1}\left(D+a_{1}\right)\right) .
$$

Then, $S S 3=\left(s_{0}^{i n}, 0, M_{1}\left(D+a_{1}\right), \frac{D}{D+a_{1}}\left(s_{1}^{i n}-M_{1}\left(D+a_{1}\right)\right)\right)$. It exists if and only if $s_{1}^{i n}>M_{1}\left(D+a_{1}\right)$.

Proof of Proposition 4.1. The local stability of each steady state depends on the sign of the real parts of the eigenvalues of the corresponding Jacobian matrix. At a given steady state $\left(s_{0}, x_{0}, s_{1}, x_{1}\right)$, this matrix is given by:

$$
J=\left[\begin{array}{cccc}
-D-E x_{0} & -\mu_{0} & F x_{0} & 0 \\
E x_{0} & \mu_{0}-D-a_{0} & -F x_{0} & 0 \\
E x_{0} & \mu_{0} & -D-F x_{0}-G x_{1} & -\mu_{1} \\
0 & 0 & G x_{1} & \mu_{1}-D-a_{1}
\end{array}\right]
$$

where

$$
E=\frac{\partial \mu_{0}}{\partial s_{0}}\left(s_{0}, s_{1}\right)>0, \quad F=-\frac{\partial \mu_{0}}{\partial s_{1}}\left(s_{0}, s_{1}\right)>0, \quad G=\frac{d \mu_{1}}{d s_{1}}\left(s_{1}\right)>0
$$

The eigenvalues of (A.2) are the roots of its characteristic polynomial $\operatorname{det}(J-\lambda I)$. Notice that we have used the opposite sign for the partial derivative $F=-\frac{\partial \mu_{0}}{\partial s_{1}}\left(s_{0}, s_{1}\right)$, so that all constants involved in the computations become positive, which will simplify the analysis of the characteristic polynomial of (A.2).

- For $S S 0=\left(s_{0}^{i n}, 0, s_{1}^{i n}, 0\right)$, the Jacobian matrix (A.2) reads

$$
J=\left[\begin{array}{cccc}
-D & -\mu_{0}\left(s_{0}^{i n}, s_{1}^{i n}\right) & 0 & 0 \\
0 & \mu_{0}\left(s_{0}^{i n}, s_{1}^{i n}\right)-D-a_{0} & 0 & 0 \\
0 & \mu_{0}\left(s_{0}^{i n}, s_{1}^{i n}\right) & -D & -\mu_{1}\left(s_{1}^{i n}\right) \\
0 & 0 & 0 & \mu_{1}\left(s_{1}^{i n}\right)-D-a_{1}
\end{array}\right] .
$$

Its eigenvalues are $\lambda_{1}=\mu_{0}\left(s_{0}^{i n}, s_{1}^{i n}\right)-D-a_{0}, \lambda_{2}=\mu_{1}\left(s_{1}^{i n}\right)-D-a_{1}$ and $\lambda_{3}=\lambda_{4}=-D$. For being stable, we need $\lambda_{1}<0$ and $\lambda_{2}<0$. Therefore, SS0 is stable if and only if

$$
\mu_{0}\left(s_{0}^{i n}, s_{1}^{i n}\right)<D+a_{0}
$$

and

$$
\mu_{1}\left(s_{1}^{i n}\right)<D+a_{1}
$$

For $s_{1}$ fixed, since the function $s_{0} \mapsto \mu_{0}\left(s_{0}, s_{1}\right)$ is increasing, we have the following equivalence:

$$
\mu_{0}\left(s_{0}^{i n}, s_{1}^{i n}\right)<D+a_{0} \Longleftrightarrow s_{0}^{i n}<M_{0}\left(D+a_{0}, s_{1}^{i n}\right) .
$$


The function $s_{1} \mapsto \mu_{1}\left(s_{1}\right)$ is increasing, then we have:

$$
\mu_{1}\left(s_{1}^{i n}\right)<D+a_{1} \Longleftrightarrow s_{1}^{i n}<M_{1}\left(D+a_{1}\right) .
$$

Therefore, $S S 0$ is locally exponentially stable if and only if $s_{1}^{i n}<M_{1}\left(D+a_{1}\right)$ and $s_{0}^{i n}<M_{0}\left(D+a_{0}, s_{1}^{i n}\right)$.

- For $S S 1=\left(s_{01}, x_{01}, s_{11}, 0\right)$, where $s_{01}$ is the solution of the equation: $\mu_{0}\left(s_{01},\left(s_{0}^{\text {in }}+s_{1}^{\text {in }}\right)-s_{01}\right)=D+a_{0}$, $x_{01}=\frac{D}{D+a_{0}}\left(s_{0}^{i n}-s_{01}\right)$ and $s_{11}=\left(s_{0}^{i n}+s_{1}^{i n}\right)-s_{01}$, the Jacobian matrix (A.2) becomes:

$$
J=\left[\begin{array}{cccc}
-D-E x_{0} & -D-a_{0} & F x_{0} & 0 \\
E x_{0} & 0 & -F x_{0} & 0 \\
E x_{0} & D+a_{0} & -D-F x_{0} & -\mu_{1} \\
0 & 0 & 0 & \mu_{1}-D-a_{1}
\end{array}\right]
$$

Its characteristic polynomial is:

$$
\operatorname{det}(J-\lambda I)=\left(\lambda-\mu_{1}+D+a_{1}\right)(\lambda+D)\left(\lambda^{2}+\left[D+(E+F) x_{0}\right] \lambda+\left(D+a_{0}\right)(E+F) x_{0}\right)
$$

Its eigenvalues are $\lambda_{1}=\mu_{1}-D-a_{1}, \lambda_{2}=-D$ and $\lambda_{3}$ and $\lambda_{4}$ are the roots of the following quadratic equation:

$$
\lambda^{2}+\left[D+(E+F) x_{0}\right] \lambda+\left(D+a_{0}\right)(E+F) x_{0}=0
$$

Since $\lambda_{3} \lambda_{4}=\left(D+a_{0}\right)(E+F) x_{0}>0$ and $\lambda_{3}+\lambda_{4}=-\left[D+(E+F) x_{0}\right]<0$, the real parts of $\lambda_{3}$ and $\lambda_{4}$ are negative. So, for being stable we must have $\lambda_{1}<0$. Therefore, SS1 is stable if and only if

$$
\mu_{1}\left(s_{0}^{i n}+s_{1}^{i n}-s_{0}\right)<D+a_{1}
$$

where $s_{0}$ is the solution of $\mu_{0}\left(s_{0},\left(s_{0}^{i n}+s_{1}^{i n}\right)-s_{0}\right)=D+a_{0}$. Since the function $s_{1} \mapsto \mu_{1}\left(s_{1}\right)$ is increasing, we have the following equivalence

$$
\mu_{1}\left(s_{0}^{i n}+s_{1}^{i n}-s_{0}\right)<D+a_{1} \Longleftrightarrow s_{0}>s_{0}^{i n}+s_{1}^{i n}-M_{1}\left(D+a_{1}\right) .
$$

Since the function $s_{0} \mapsto \psi\left(s_{0}\right)=\mu_{0}\left(s_{0}, s_{0}^{i n}+s_{1}^{i n}-s_{0}\right)$ is increasing, we deduce that $\psi\left(s_{0}\right)>$ $\psi\left(s_{0}^{i n}+s_{1}^{i n}-M_{1}\left(D+a_{1}\right)\right)$. Since,

$$
\psi\left(s_{0}\right)=\mu_{0}\left(s_{0}, s_{0}^{i n}+s_{1}^{i n}-s_{0}\right)=D+a_{0}
$$

Therefore, the condition $\mu_{1}\left(s_{0}^{i n}+s_{1}^{i n}-s_{0}\right)<D+a_{1}$ of stability of SS1 is equivalent to:

$$
D+a_{0}>\mu_{0}\left(s_{0}^{i n}+s_{1}^{i n}-M_{1}\left(D+a_{1}\right), M_{1}\left(D+a_{1}\right)\right) .
$$

Since the function $s_{0} \mapsto \mu_{0}\left(s_{0}, M_{1}\left(D+a_{1}\right)\right)$ is increasing, the condition $D+a_{0}>\mu_{0}\left(s_{0}^{i n}+s_{1}^{i n}-M_{1}(D+\right.$ $\left.\left.a_{1}\right), M_{1}\left(D+a_{1}\right)\right)$ is equivalent to

$$
s_{0}^{i n}+s_{1}^{i n}-M_{1}\left(D+a_{1}\right)<M_{0}\left(D+a_{0}, M_{1}\left(D+a_{1}\right)\right),
$$

which is equivalent to

$$
s_{0}^{i n}+s_{1}^{i n}<M_{1}\left(D+a_{1}\right)+M_{0}\left(D+a_{0}, M_{1}\left(D+a_{1}\right)\right) .
$$


Therefore, $S S 1$ is locally exponentially stable if and only if $s_{0}^{i n}+s_{1}^{i n}<M_{0}\left(D+a_{0}, M_{1}\left(D+a_{1}\right)\right)+M_{1}$ $\left(D+a_{1}\right)$.

- For $S S 2=\left(s_{02}, x_{02}, s_{12}, x_{12}\right)$, where $s_{02}=M_{0}\left(D+a_{0}, M_{1}\left(D+a_{1}\right)\right), x_{02}=\frac{D}{D+a_{0}}\left(s_{0}^{i n}-s_{02}\right), s_{12}=$ $M_{1}\left(D+a_{1}\right)$ and $x_{12}=\frac{D}{D+a_{1}}\left(\left(s_{0}^{i n}+s_{1}^{i n}\right)-s_{02}-s_{12}\right)$.

At SS2, the Jacobian matrix is given by:

$$
J=\left[\begin{array}{cccc}
-D-E x_{0} & -D-a_{0} & F x_{0} & 0 \\
E x_{0} & 0 & -F x_{0} & 0 \\
E x_{0} & D+a_{0} & -D-F x_{0}-G x_{1} & -D-a_{1} \\
0 & 0 & G x_{1} & 0
\end{array}\right]
$$

Its characteristic polynomial is:

$$
\operatorname{det}(J-\lambda I)=\lambda^{4}+f_{1} \lambda^{3}+f_{2} \lambda^{2}+f_{3} \lambda+f_{4},
$$

where

$$
\begin{aligned}
& f_{1}=G x_{1}+(E+F) x_{0}+2 D, \\
& f_{2}=E G x_{0} x_{1}+\left(2 D+a_{0}\right)(E+F) x_{0}+\left(2 D+a_{1}\right) G x_{1}+D^{2}, \\
& f_{3}=\left(2 D+a_{0}+a_{1}\right) E G x_{0} x_{1}+D\left(D+a_{0}\right)(E+F) x_{0}+D\left(D+a_{1}\right) G x_{1}, \\
& f_{4}=\left(D+a_{0}\right)\left(D+a_{1}\right) E G x_{0} x_{1} .
\end{aligned}
$$

We use the Routh-Hurwitz criterium for the stability of $S S 2$. Using the same arguments as Appendix D [5], we have:

$$
\begin{aligned}
& f_{i}>0 \text { for } i=1, \ldots, 4, \\
& f_{1} f_{2}-f_{3}>0, \\
& f_{1} f_{2} f_{3}-f_{1}^{2} f_{4}-f_{3}^{2}>0 .
\end{aligned}
$$

According to (A.3), (A.4) and (A.5) the Routh-Hurwitz criteria are satisfied. Therefore, SS2 is stable if and only if $x_{0}=x_{02}>0$ and $x_{1}=x_{12}>0$. This means that $s_{0}^{i n}>M_{0}\left(D+a_{0}, M_{1}\left(D+a_{1}\right)\right)$ and $s_{0}^{i n}+s_{1}^{i n}>M_{0}\left(D+a_{0}, M_{1}\left(D+a_{1}\right)\right)+M_{1}\left(D+a_{1}\right)$. Therefore, SS2 is stable as long as it exists.

- For SS3 $=\left(s_{0}^{i n}, 0, M_{1}\left(D+a_{1}\right), \frac{D}{D+a_{1}}\left(s_{1}^{i n}-M_{1}\left(D+a_{1}\right)\right)\right)$, the Jacobian matrix (A.2) becomes

$$
J=\left[\begin{array}{cccc}
-D & -\mu_{0} & 0 & 0 \\
0 & \mu_{0}-D-a_{0} & 0 & 0 \\
0 & \mu_{0} & -D-G x_{1} & -D-a_{1} \\
0 & 0 & G x_{1} & 0
\end{array}\right]
$$

Its characteristic polynomial is:

$$
\operatorname{det}(J-\lambda I)=(-D-\lambda)\left(\mu_{0}-D-a_{0}-\lambda\right)\left(\left[D+G x_{1}+\lambda\right] \lambda+\left(D+a_{1}\right) G x_{1}\right) .
$$

Its eigenvalues are $\lambda_{1}=-D, \lambda_{2}=\mu_{0}-D-a_{0}$ and $\lambda_{3}$ and $\lambda_{4}$ are the roots of the following quadratic equation:

$$
\lambda^{2}+\left[D+G x_{1}\right] \lambda+\left(D+a_{1}\right) G x_{1}=0 .
$$


Since $\lambda_{3} \lambda_{4}=\left(D+a_{1}\right) G x_{1}>0$ and $\lambda_{3}+\lambda_{4}=-\left(D+G x_{1}\right)<0$, the real parts of $\lambda_{3}$ and $\lambda_{4}$ are negative. Therefore, SS3 is locally exponentially stable if and only if $\lambda_{2}<0$, that is to say

$$
\mu_{0}\left(s_{0}^{i n}, M_{1}\left(D+a_{1}\right)\right)<D+a_{0}
$$

which is equivalent to

$$
s_{0}^{\text {in }}<M_{0}\left(D+a_{0}, M_{1}\left(D+a_{1}\right)\right)
$$

Proof of Proposition 4.2. $F_{0}$ is defined if and only if $M_{0}\left(D+a_{0}, s_{1}^{i n}\right)$ is defined. This means that, $D+a_{0}<$ $m_{0}\left(s_{1}^{\text {in }}\right)$ which is equivalent to $D<m_{0}\left(s_{1}^{i n}\right)-a_{0}=D_{0}$.

$F_{1}$ is defined if and only if $M_{1}\left(D+a_{1}\right)$ and $M_{0}\left(D+a_{0}, M_{1}\left(D+a_{1}\right)\right)$ are defined. This means that, $D+a_{1}<$ $m_{1}$ and $D+a_{0}<m_{0}\left(M_{1}\left(D+a_{1}\right)\right)$. Since the function $D \longmapsto m_{0}\left(M_{1}\left(D+a_{1}\right)\right)$ is decreasing then $m_{0}\left(M_{1}(D+\right.$ $\left.\left.a_{1}\right)\right)<m_{0}\left(M_{1}\left(a_{1}\right)\right) . D+a_{0}<m_{0}\left(M_{1}\left(D+a_{1}\right)\right)$ is satisfied if and only if $D<D_{2}$ where $D_{2}$ is the positive solution, if it exists, of $D+a_{0}=m_{0}\left(M_{1}\left(D+a_{1}\right)\right)$. The solution $D_{2} \geq 0$ exists if and only if $a_{0}<m_{0}\left(M_{1}\left(a_{1}\right)\right)$. Thus, $F_{1}$ is defined on $\left[0, \min \left(m_{1}-a_{1}, D_{2}\right)[\right.$.

By the same way, $F_{2}$ is defined on $\left[0, D_{2}[\right.$ when it exists.

The idea of the proof of Theorem 5.2 is based on Lemma 5.1 and comparison between the growth functions. We first prove Lemma 5.1.

\section{Proof of Lemma 5.1.}

- If we have $\mu_{1}\left(s_{1}^{i n}\right)<a_{1}<D+a_{1}$ then $s_{1}^{i n}<M_{1}\left(D+a_{1}\right) . M_{0}$ is increasing with respect to the second variable then $M_{0}\left(D+a_{0}, s_{1}^{\text {in }}\right)<M_{0}\left(D+a_{0}, M_{1}\left(D+a_{1}\right)\right)$. By using $s_{1}^{\text {in }}<M_{1}$ $\left(D+a_{1}\right)$, which is equivalent to $M_{1}\left(D+a_{1}\right)-s_{1}^{i n}>0$, we obtain

$$
M_{0}\left(D+a_{0}, s_{1}^{i n}\right)<M_{0}\left(D+a_{0}, M_{1}\left(D+a_{1}\right)\right)+M_{1}\left(D+a_{1}\right)-s_{1}^{i n} .
$$

Therefore, $F_{0}(D)<F_{1}(D)-s_{1}^{\text {in }}$, for all $D>0$.

- If we have $\mu_{1}\left(s_{1}^{\text {in }}\right)>a_{1}$ and $D>\bar{D}=\mu_{1}\left(s_{1}^{i n}\right)-a_{1} \Leftrightarrow \mu_{1}\left(s_{1}^{i n}\right)<D+a_{1}$. Then, $s_{1}^{i n}<M_{1}\left(D+a_{1}\right)$ and $M_{0}\left(D+a_{0}, s_{1}^{\text {in }}\right)<M_{0}\left(D+a_{0}, M_{1}\left(D+a_{1}\right)\right)$. Since $M_{1}\left(D+a_{1}\right)>s_{1}^{\text {in }}$, we obtain

$$
M_{0}\left(D+a_{0}, s_{1}^{i n}\right)<M_{0}\left(D+a_{0}, M_{1}\left(D+a_{1}\right)\right)+M_{1}\left(D+a_{1}\right)-s_{1}^{i n} .
$$

Therefore, $F_{0}(D)<F_{1}(D)-s_{1}^{\text {in }}$, for all $D>0$.

- If we have $\mu_{1}\left(s_{1}^{i n}\right)>a_{1}$ and $D<\bar{D}=\mu_{1}\left(s_{1}^{i n}\right)-a_{1} \Leftrightarrow M_{1}\left(D+a_{1}\right)<s_{1}^{i n}$. Then, we have

$$
M_{0}\left(D+a_{0}, M_{1}\left(D+a_{1}\right)\right)<M_{0}\left(D+a_{0}, s_{1}^{i n}\right) .
$$

Therefore, $F_{2}(D)<F_{0}(D)$, for all $D>0$. 
Proof of Theorem 5.2. Theorem 5.2 follows from Lemma 5.1 and the next inequalities:

- If $\mu_{1}\left(s_{1}^{i n}\right)<a_{1}$ and $F_{1}(D)-s_{1}^{i n}<s_{0}^{\text {in }}$ then $F_{2}(D)<s_{0}^{\text {in }}$, for all $D>0$.

Indeed, if $\mu_{1}\left(s_{1}^{i n}\right)<a_{1}$ then $M_{1}\left(D+a_{1}\right)-s_{1}^{\text {in }}>0$. Then,

$$
M_{0}\left(D+a_{0}, M_{1}\left(D+a_{1}\right)\right)<M_{0}\left(D+a_{0}, M_{1}\left(D+a_{1}\right)\right)+M_{1}\left(D+a_{1}\right)-s_{1}^{i n}=F_{1}(D)-s_{1}^{i n} .
$$

Since $F_{1}(D)-s_{1}^{\text {in }}<s_{0}^{i n}$ then $F_{2}(D)<s_{0}^{i n}$.

- If $\mu_{1}\left(s_{1}^{i n}\right)>a_{1}, D>\bar{D}$ and $F_{1}(D)-s_{1}^{\text {in }}<s_{0}^{i n}$ then $F_{2}(D)<s_{0}^{\text {in }}$, for all $D>0$.

Indeed, $D>\bar{D} \Leftrightarrow \mu_{1}\left(s_{1}^{i n}\right)<D+a_{1} \Leftrightarrow M_{1}\left(D+a_{1}\right)-s_{1}^{i n}>0$. Then,

$$
M_{0}\left(D+a_{0}, M_{1}\left(D+a_{1}\right)\right)<M_{0}\left(D+a_{0}, M_{1}\left(D+a_{1}\right)\right)+M_{1}\left(D+a_{1}\right)-s_{1}^{i n}=F_{1}(D)-s_{1}^{i n} .
$$

Finally, $F_{1}(D)-s_{1}^{\text {in }}<s_{0}^{\text {in }}$ implies that $F_{2}(D)<s_{0}^{\text {in }}$.

- If $\mu_{1}\left(s_{1}^{\text {in }}\right)>a_{1}, D<\bar{D}$ and $F_{0}(D)<s_{0}^{\text {in }}$ then $F_{1}(D)-s_{1}^{i n}<s_{0}^{i n}$, for all $D>0$.

Indeed, $D<\bar{D}=\mu_{1}\left(s_{1}^{i n}\right)-a_{1} \Leftrightarrow M_{1}\left(D+a_{1}\right)<s_{1}^{i n}$. Since $F_{0}(D)=M_{0}\left(D+a_{0}, s_{1}^{i n}\right)<s_{0}^{i n}$, then

$$
F_{1}(D)-s_{1}^{i n}=M_{0}\left(D+a_{0}, M_{1}\left(D+a_{1}\right)\right)+M_{1}\left(D+a_{1}\right)-s_{1}^{i n}<M_{0}\left(D+a_{0}, s_{1}^{i n}\right),
$$

which implies that $F_{1}(D)-s_{1}^{i n}<s_{0}^{i n}$.

- If $\mu_{1}\left(s_{1}^{i n}\right)>a_{1}, D<\bar{D}$ and $F_{2}(D)<s_{0}^{\text {in }}$ then $F_{1}(D)-s_{1}^{\text {in }}<s_{0}^{\text {in }}$, for all $D>0$.

Indeed, $D<\bar{D}=\mu_{1}\left(s_{1}^{\text {in }}\right)-a_{1} \Leftrightarrow M_{1}\left(D+a_{1}\right)<s_{1}^{\text {in }}$. Since $F_{2}(D)=M_{0}\left(D+a_{0}, M_{1}\left(D+a_{1}\right)\right)<s_{0}^{i n}$, then

$$
F_{1}(D)-s_{1}^{i n}=M_{0}\left(D+a_{0}, M_{1}\left(D+a_{1}\right)\right)+M_{1}\left(D+a_{1}\right)-s_{1}^{i n}<M_{0}\left(D+a_{0}, M_{1}\left(D+a_{1}\right)\right) .
$$

Finally, we obtain

$$
F_{1}(D)-s_{1}^{i n}<s_{0}^{i n}
$$

The proof of Theorem 5.4 is based on Lemma 5.3 and comparison between growth functions. We first prove Lemma 5.3.

\section{Proof of Lemma 5.3.}

- If $D>\overline{D_{1}}$ then $F_{2}(D)>F_{2}\left(\bar{D}_{1}\right)=s_{0}^{i n}$ and we obtain

$$
F_{1}(D)-s_{0}^{i n}>F_{1}(D)-F_{2}(D) .
$$

In the other hand, we have $M_{0}\left(D+a_{0}, M_{1}\left(D+a_{1}\right)\right)>s_{0}^{\text {in }}$. Since $\mu_{0}$ is increasing with respect to the first variable then $D+a_{0}>\mu_{0}\left(s_{0}^{i n}, M_{1}\left(D+a_{1}\right)\right)$. $M_{2}$ is decreasing with respect to the second variable then $M_{2}\left(s_{0}^{i n}, D+a_{0}\right)<M_{1}\left(D+a_{1}\right)$. Finally, we obtain

$$
F_{1}(D)-F_{2}(D)>F_{3}(D)
$$

- If $D<\overline{D_{1}}$ and $\overline{D_{1}}>0$, then $F_{2}(D)<F_{2}\left(\overline{D_{1}}\right)=s_{0}^{\text {in }}$ and we obtain

$$
F_{1}(D)-s_{0}^{i n}<F_{1}(D)-F_{2}(D) .
$$


Now, $M_{0}\left(D+a_{0}, M_{1}\left(D+a_{1}\right)\right)<s_{0}^{i n}$ implies that $D+a_{0}<\mu_{0}\left(s_{0}^{i n}, M_{1}\left(D+a_{1}\right)\right) . M_{2}$ is decreasing with respect to the second variable then $M_{2}\left(s_{0}^{\text {in }}, D+a_{0}\right)>M_{1}\left(D+a_{1}\right)$. Finally, we obtain

$$
F_{1}(D)-F_{2}(D)<F_{3}(D) \text {. }
$$

- We have $F_{2}\left(\bar{D}_{1}\right)=s_{0}^{\text {in }}$ then $F_{1}\left(\bar{D}_{1}\right)-F_{2}\left(\bar{D}_{1}\right)=F_{1}\left(\bar{D}_{1}\right)-s_{0}^{\text {in }}$. This implies that $F_{1}-F_{2}$ and $F_{1}-s_{0}^{\text {in }}$ intersect at the value $D=\overline{D_{1}}$. On the other hand, $F_{2}\left(\bar{D}_{1}\right)=s_{0}^{\text {in }}$ is equivalent to $M_{0}\left(\bar{D}_{1}+a_{0}, M_{1}\left(\bar{D}_{1}+\right.\right.$ $\left.\left.a_{1}\right)\right)=s_{0}^{\text {in }}$. Then, we have $\mu_{0}\left(s_{0}^{i n}, M_{1}\left(\bar{D}_{1}+a_{1}\right)\right)=\bar{D}_{1}+a_{0}$. Now, $F_{3}\left(\bar{D}_{1}\right)=M_{2}\left(s_{0}^{i n}, \bar{D}_{1}+a_{0}\right)$ which is equivalent to $\mu_{0}\left(s_{0}^{\text {in }}, F_{3}\left(\bar{D}_{1}\right)\right)=\bar{D}_{1}+a_{0}$. The two last equalities lead to $F_{3}\left(\bar{D}_{1}\right)=M_{1}\left(\bar{D}_{1}+a_{1}\right)$. Thus, $F_{3}\left(\bar{D}_{1}\right)=F_{1}\left(\bar{D}_{1}\right)-F_{2}\left(\bar{D}_{1}\right)=F_{1}\left(\bar{D}_{1}\right)-s_{0}^{\text {in }}$. Consequently, $F_{3}, F_{1}-F_{2}$ and $F_{1}-s_{0}^{\text {in }}$ intersect at $D=\bar{D}_{1}$. Since $F_{3}$ is decreasing, then $\bar{D}_{3}=\sup _{D} F_{3}(D)$ then $\bar{D}_{3}>\bar{D}_{1}$.

Proof of Theorem 5.4. Theorem 5.4 is a consequence of Lemma 5.3. Notice that if $\bar{D}_{3}<0$ then $F_{3}(D)<0$ and since $\bar{D}_{1}<\bar{D}_{3}$, the case $\bar{D}_{1}>0$ and $\bar{D}_{3}<0$ cannot occur.

Acknowledgements. The authors thank the Euromed $3+3$ project TREASURE (http://www.inra.fr/treasure) and the project PHC- UTIQUE $13 G 1120$ who partly financed this research.

\section{REFERENCES}

[1] A. Burchard, Substrate degradation by a mutualistic association of two species in the chemostat. J. Math. Biol. 32 (1994) $465-489$.

[2] M. El Hajji, F. Mazenc and J. Harmand, A mathematical study of a syntrophic relationship of a model of anaerobic digestion process. Math. Biosci. Eng. 7 (2010) 641-656.

[3] R. Kreikenbohm and E. Bohl, A mathematical model of syntrophic cocultures in the chemostat. FEMS Microbiol. Ecol. 38 (1986) 131-140.

[4] R. Kreikenbohm and E. Bohl, Bistability in the chemostat. Ecol. Model. 43 (1988) $287-301$.

[5] T. Sari and J. Harmand, A model of a syntrophic relationship between two microbial species in a chemostat including maintenance. Math. Biosci. Eng. 275 (2016) 1-9.

[6] T. Sari, M. El Hajji and J. Harmand, The mathematical analysis of a syntrophic relationship between two microbial species in a chemostat. Math. Biosci. Eng. 9 (2012) 627-645.

[7] G. Stephanopoulos, The dynamics of commensalism. Biotechnol. Bioeng. 23 (1981) 2243-2255.

[8] E.I.P. Volcke, M. Sbarciog, E.J.L. Noldus, B. De Baets and M. Loccufier, steady state multiplicity of two-step biological conversion systems with general kinetics. Math. Biosci. 228 (2010) 160-170.

[9] M.J. Wade, R.W. Pattinson, N.G. Parker and J. Dolfing, Emergent behaviour in a chlorophenol-mineralising three-tired microbial "food web". Theor. Biol. 389 (2016) 171-186.

[10] T.G. Wilkinson, H.H. Topiwala and G. Hamer, Interactions in a mixed bacterial population growing on methane in continuous culture. Biotechnol. Bioeng. 16 (1974) 41-59.

[11] A. Xu, J. Dolfing, T.P. Curtis, G. Montague and E. Martin, Maintenance affects the stability of a two-tiered microbial "food chain". J. Theor. Biol. 276 (2011) 35-41. 